\title{
La telecardiologia come strumento di integrazione tra ospedale e territorio
}

\author{
Selezione dei contributi al Simposio ANMCO \\ Regione Lombardia, tenutosi a Milano l'8 ottobre 2005
}

Monaldi Arch Chest Dis 2005; 64: 134-150.

\section{INTRODUZIONE}

Da circa un decennio la cardiologia rappresenta uno degli ambiti disciplinari privilegiati per la sperimentazione e la validazione delle applicazioni della telemedicina. Il contesto clinico delle patologie cardiovascolari più diffuse, caratterizzato dalla necessità di trasferire rapidamente segnali biologici e parametri quantitativi e di disporre, altrettanto rapidamente, di interpretazioni ed indicazioni sui nodi decisionali più critici per una corretta ed efficace gestione delle emergenza, hanno rappresentato il terreno ideale su cui cimentare le tecnologie di telemedicina.

Tuttavia, proprio questi aspetti hanno contribuito a polarizzare l'attenzione dei cardiologi e dei medici in generale su questi versanti della telecardiologia più strettamente connessi alla tecnologia, piuttosto che sui contenuti e sulle ricadute di tipo clinico e organizzativo indispensabili per una tecnica che aspira ad assumere una posizione di rilievo nei processi di cura.

L'interesse per la telecardiologia è confermato dal numero di esperienze che anche in Italia si sono sviluppate su questo argomento. Purtroppo la frammentazione di queste esperienze, che ha avuto come esito una serie di studi riferiti per lo più a casistiche molto ristrette, ha limitato fortemente fino ad oggi (con qualche importante eccezione) una significativa diffusione delle applicazioni di telecardiologia in scenari clinici reali. A questa situazione si deve anche il fatto che, ad oggi, al di fuori di sperimenta- zioni, nessuna Regione ha predisposto delle ipotesi di tariffazione per i servizi di telecardiologia.

Importante è stata quindi l'intuizione di Soccorso Capomolla e di Antonio Mafrici di organizzare, come Sezione Lombarda dell'AMNCO, un simposio dedicato alla telecardiologia come strumento di integrazione tra ospedale e territorio. Al disegno del simposio, tenutosi a Milano l'8 ottobre 2005, è stato dato un taglio molto pratico, affidando la trattazione dei vari argomenti ai gruppi che sicuramente in Italia hanno maturato le esperienze più consistenti.

Non a caso lo scompenso cardiaco cronico e la gestione domiciliare del paziente post-acuto sono stati individuati come gli ambiti di applicazione di maggior interesse sul piano clinico, organizzativo ed economico. La loro diretta attinenza con la cardiologia riabilitativa sta alla base della scelta editoriale di pubblicare sugli Archives una selezione dei contributi dei Relatori invitati.

La differenza del format dei singoli contributi rispecchia il carattere seminariale dell'evento e la multiprofessionalità (cardiologo, infermiere professionale, bio-ingegnere, informatico) che caratterizza la composizione delle équipe che lavorano in realtà in cui la telemedicina, superata la fase di sperimentazione, rappresenta già un modello innovativo di cura.

\section{ROBERTO TRAMARIN}

Fondazione Europea di Ricerca Biomedica Cernusco S/N, Milano (I) 


\section{IL SERVIZIO DI TELEMONITORAGGIO}

Soccorso Capomolla, GianDomenico Pinna, Roberto Maestri, Marina Ferrari, Monica Ceresa

IRCCS Fondazione Salvatore Maugeri

- Istituto Scientifico Montescano, Montescano (PV), Italia E-mail: scapomolla@fsm.it

\section{Introduzione}

Lo scompenso cardiaco cronico (SCC) è una sindrome che determina una riduzione della capacità funzionale, una cattiva qualità della vita ed una ridotta sopravvivenza. La sua incidenza e prevalenza sono nettamente aumentate nei paesi industrializzati. ${ }^{1}$ Le cause di quest'aumento vanno ricercate, da una parte nell'incremento dell'età media della popolazione e dall'altra nella migliore gestione in fase acuta delle patologie condizionanti tale sindrome clinica. ${ }^{2}$

Negli Stati Uniti è stato valutato che circa tre milioni d'individui sono affetti da SCC, mentre in Italia lo studio SEOSI ha dimostrato come, mediamente, ogni anno nei reparti cardiologici siano ricoverati circa 65000 pazienti con scompenso cardiaco cronico. ${ }^{3}$ Per altro, questi ultimi rappresentano solo una piccola parte dei pazienti affetti da SCC nel nostro Paese, poiché la maggioranza afferisce ad altre Divisioni Mediche o Geriatriche. Un'analisi comparativa delle SDO, tra il 1996 ed il 2001, ha evidenziato un incremento del DRG 127 del $39.5 \%$ che è diventato il terzo DRG più frequente nell'epidemiologia ospedaliera. L'analisi della valorizzazione economica, evidenzia che il DRG 127 è quello che determina il maggiore assorbimento di risorse tra i primi tre $\mathrm{DRG}$ più frequenti. 4

$\grave{E}$ inoltre noto che il $78 \%$ di pazienti con SCC subisce mediamente due ricoveri l'anno per instabilizzazione emodinamica. ${ }^{5}$ Ciò evidenzia la forte domanda sanitaria per tale patologia, alla quale è data spesso una risposta inappropriata o carente 6 con un elevato consumo annuale di risorse. ${ }^{7,8}$ Negli USA, quest'ultimo raggiunge i $18 \mathrm{mi}-$ lioni di dollari, di cui circa $2 / 3$ spesi per riospedalizzazioni causate da instabilizzazioni cliniche. ${ }^{9}$ Studi d'economia sanitaria rilevano la necessità di ridurre tale spesa contenendo soprattutto l'accesso ripetuto a strutture ospedaliere per acuti. 10

Queste considerazioni impongono quindi la necessità di ridisegnare percorsi sanitari, che possano determinare un contenimento delle risorse senza, peraltro, diminuire la qualità della prestazione sanitaria.

Allo stato attuale, in Italia, la gestione di questa patologia cronica presenta diversi punti di criticità. Il primo è legato al nuovo modo di distribuzione delle risorse: l'introduzione del pagamento per prestazione impone la gestione della fase acuta di tale sindrome, penalizzando l'approccio multidisciplinare e la continuità assistenziale che tendono a stabilizzare la fase cronica. Il secondo è correlato alla rete sanitaria: com'evidenziato recentemente dai dati di studi osservazionali quali il TEMISTOCLE e l'OSCUR solo circa il $30 \%$ di pazienti affetti afferisce in divisioni di cardiologia. ${ }^{11}$ Una recente Survey europea ha evidenziato, analogamente, come la domanda sanitaria attinente lo scompenso cardiaco sia gestita dai cardiologi solo nella misura del 43\%.12 Ciò comporta una gestione disomogenea per quanto riguarda sia i percorsi valutativi sia quelli terapeutici. In particolare i dati del data-base IN-CHF dell'ANMCO dimostrano come attualmente il percorso di stratificazione del rischio in questa popolazione, anche in ambiente cardiologico, rimanga carente. ${ }^{13}$ Infatti, solo il $65 \%$ dei pazienti ha eseguito un ecocardiogramma, e solo il $12 \%$, un test cardiopolmonare; inoltre l'utilizzo di risorse, in termini di esami strumentali più complessi, è completamente svincolato dalla gravità clinica del paziente e/o dalla pa- tologia di base della sindrome clinica. ${ }^{14}$ In Europa, solo il $63 \%$ dei pazienti ricoverati con diagnosi di scompenso ha eseguito un ecocardiogramma ed in questi gli indici di funzione sistolica erano riportati solo nel $46 \%$ dei referti. 12 Il test cardiopolmonare è stato eseguito solo in una minorità di casi. L'informazione circa il timing di tali esami nella rimodulazione del rischio cardiaco vascolare è del tutto carente. Quando si considera l'intervento terapeutico, in Italia, solo il $16 \%$ dei pazienti assume betabloccanti mentre, seppur $85 \%$ dei pazienti assume ACEInibitori, ben il 52\% l'assume a dosaggi inferiori al 50\% della dose target definite dai trias. ${ }^{11}$ In Europa la prescrizione dell'Ace-inibitore nella popolazione con scompenso cardiaco è condizionata dal grado di disfunzione ventricolare; in riferimento al trattamento beta-bloccante è stata rilevata una forte disomogeneità della prescrizione tra i diversi paesi. ${ }^{15}$ Quando, poi, si analizza l'intervento terapeutico non farmacologico è possibile rilevare come il riposo sia prescritto nel $43.5 \%$ mentre l'attività fisica consigliata solo nel $3.9 \% .^{3}$ Del resto recenti evidenze scientifiche evidenziano come i risultati dei trials spesso possano non essere francamente rappresentativi dei "vissuto quotidiano" nella gestione di questa sindrome clinica. 16 Tali distorsioni gestionali si traducono naturalmente in un alterato assorbimento delle risorse economiche dedicate con una riduzione dell'efficacia ed efficienza gestionale.

Queste considerazioni rafforzano l'esigenza strategica di costruire modelli sanitari per la gestione di questa sindrome clinica che si facciano carico non già del bisogno acuto ed emergente del singolo paziente affetto, ma di realizzare programmi fortemente integrati in termini di organizzazione e processo di cura capaci di perseguire, mediante una rete della continuità assistenziale, un intervento preventivo che governi i bisogni e la storia naturale della sindrome clinica. In letteratura sono riportate esperienze incoraggianti che mostrano come modelli a basso consumo di risorse siano efficaci nell'indurre miglioramenti del quadro funzionale e della qualità della vita con riduzione delle ospedalizzazioni e delle loro durata.

\section{La continuità assistenziale}

Nel contesto sopra definito, è necessario ripensare i determinanti del risultato che, schematicamente, possono essere identificati nell'intervento terapeutico e nella continuità assistenziale. Nonostante il netto miglioramento dell'armamentario terapeutico, nella fase attuale, tale determinante sta conoscendo una fase di nadir; la continuità assistenziale - costituisce uno snodo decisionale importante nel tentativo di ridefinizione dell'organizzazione dei processi sanitari inerenti lo scompenso cardiaco cronico. Il concetto di continuità assistenziale si struttura su due elementi specifici: 1) il diritto alla salute e 2) l'organizzazione del processo sanitario che sottende l'applicazione dei percorsi diagnostico-terapeutici capaci di contrastare l'evoluzione naturale della sindrome clinica.

In questa prospettiva la continuità assistenziale diventa un elemento strutturale di un modello di organizzazione dell'assistenza compatibile con le attuali disponibilità dei sistemi sanitari dei paesi moderni. ${ }^{17} \mathrm{Si}$ rende, quindi, necessario un sistema integrato di modelli capaci di farsi carico delle diverse fasi del bisogno, utilizzando il supporto delle tecnologie telematiche e dell'automazione. In questa dimensione, la continuità assistenziale si esercita non solo in un contesto territoriale ma in tutte le fasi della sindrome clinica e può interessare operatori e strutture diverse. Scopo di questo nostro contributo è quello di descrivere l'esperienza di un Servizio di telemonitoraggio clinico per lo scompenso, nato come provider tra diversi modelli gestionali (Unità per lo scompenso, Day Hospital per lo scompenso, territorio). 18 


\section{Disegno dello studio}

Lo studio è stato disegnato come uno studio randomizzato in cieco. Il paziente, dopo ottimizzazione terapeutica e stabilizzazione clinica, era randomizzato in cieco con un rapporto 1:2 in due gruppi: il primo gruppo veniva riaffidato alla gestione territoriale; il secondo gruppo al servizio di telemonitoraggio clinico. A quest'ultimo, dopo un intervento educazionale strutturato, si consegnava un calendario di accesso individualizzato sulla base di uno score di rischio per eventi cardiovascolari costruito su una popolazione di riferimento precedentemente gestita nel nostro centro. Lo score identifica tre livelli di rischio con relativa tempistica di accesso: basso rischio: il paziente accedeva al servizio ogni 60 giorni; medio rischio: l'accesso era schedulato a $30 \mathrm{gg}$; alto rischio: il paziente accedeva ogni 15 giorni. Oltre al calendario prestabilito, il paziente poteva accedere al servizio in modo estemporaneo mediante uso di una segreteria telefonica. Per ogni accesso preventivato il paziente, mediante uso della tastiera telefonica, inviava una serie di parametri vitali e di informazioni cliniche.

La fase di arruolamento è durata 36 mesi con un follow-up di 12 mesi per tutti i pazienti. L'end-point primario dello studio era rappresentato dalle reospedalizzazioni per causa cardiaca. Sono stati considerati come endpoint secondari l'aderenza al sistema e il management gestionale.

\section{Sistema di telemonitoraggio}

Il sistema di telemonitoraggio è costituito da una piattaforma progettata dal Servizio di Bioingegneria Clinica del centro di Montescano che comprende tre software gestionali: 1) L'IVR (Interactive Voice Responder costruito dalla ditta Apple elettronica srl, Torino) che consente in modo automatico la raccolta di parametri vitali ed di informazioni cliniche inviate via telefono dal paziente; 2) L'IMAC (sIstema di Monitoraggio pArametri Clinici) che consente la gestione delle chiamate, l'archiviazione dei parametri vitali, la valutazione della chiamata corrente con i limiti di confidenza per la stabilità clinica determinati individualmente; queste caratteristiche consentono una completa autonomizzazione del personale infermieristico che attiva l'intervento medico solo in caso di parametri fuori dal range di stabilità clinica; 3) la cartella clinica informatizzata che consente l'archiviazione dei dati clinici e dei parametri strumentali e vitali del paziente. L'accesso del paziente al servizio avviene mediante digitazione di un numero verde e l'inserimento di un codice personale di sicurezza. Il servizio è annesso al day-hospital ed è gestito dal personale infermieristico e medico.

\section{Risultati preliminari}

Sono stati arruolati 290 pazienti tra dicembre 2000 e dicembre 2004; il follow-up è stato completato nel dicembre 2004. Nel braccio usual care sono stati arruolati 100 pazienti, mentre il gruppo affidato al telemonitoraggio era costituito da 190 pazienti; 10 pazienti affidati a tale braccio avevano abbandonato lo studio nella fase educazionale per l'uso del sistema. Gli accessi programmati sono stati 2496, quelli realmente effettuali 2147 con una aderenza al sistema (86\%). Oltre alle chiamate programmate sono stati eseguiti 999 accessi non programmati. In $68 / 2303(0.3 \%)$ è stato riscontrato un errore di digitazione mentre non sono stati riscontrati errori nel trasferimento dei dati. Degli accessi eseguiti nel 69\% è stato dato un giudizio di stabilità clinica, mente nel $31 \%$ è stata riscontrata instabilità con la necessità di una riattivazione del processo di cura (integrazione di terapia, rimodulazioni terapeutiche, esecuzione di esami bioumorali e/o strumentali, contatto del medico di medicina generale). Le re-ospedalizzazioni per causa cardiaca sono state significativamente minori nel gruppo affidato al servizio di telemonitoraggio: $65 / 159$ (41\%) vs. $94 / 159$ $(59 \%) ; \mathrm{p}<0.007$.

\section{Conclusioni}

La continuità assistenziale è un bisogno organizzativo/gestionale finalizzato a dare una risposta appropriata alla domanda sanitaria crescente nei pazienti con scompenso cardiaco cronico. Il servizio di telemonitoraggio clinico come provider remoto a governo infermieristico e come supporto delle decisioni mediche si è dimostrato un modello gestionale sicuro, applicabile ed efficace per impattare la morbilità dello scompenso cardiaco. Quale sia il suo ruolo nella rete assistenziale, e rispetto a modelli diversi, per intensità e modalità gestionale, rimane ancora da indagare.

\section{Bibliografia}

1. Massie BM, Shah N. The heart failure epidemic: magnitude of the problem and potential mitigating approaches. Current Opinion in Cardiology 1996;11:221-226.

2. Sharpe N, Doughty R. Epidemiology of heart failure and ventricular dysfunction. Lancet 1998; 352 (suppl. I): 3-7.

3. SEOSI Investigators. Survey on heart failure in Italian hospital cardiology units. Results of the SEOSI study. Eur Heart J 1997: 18: $1457-64$.

4. http://www.ministerosalute.it/

5. Konstam MA, Dracup K, Baker DW. Heart Failure: Evaluation and care of patients with left ventricular dysfunction. Clinical practice Guideline $\mathrm{N}^{\circ}$ 11: MD department of Health and Human services, Public Healt Service, Agency for Health Care policy and Research and the National Heart, lung and Blood institute. AHCPR publication 94-0612, June 1994.

6. Erhardt LR, Cline CM. Organisation of the care of patients with heart failure. Lancet 1998; 352 (suppl I): 15-18.

7. Haldeman GA, Croft JB, Giles WH, Rashidee A. Hospitalization of patients with heart failure: National Hospital Discharge survey, 1985 to 1995 . Am Heart J 1999; 137: 352-60.

8. Steinwachs DM, Collins-Nakai RL, Cohn L, Garson A, Wolk MJ The future of Cardiology: utilization and costs of care. J Am Coll Cardiol 2000; 35 (Suppl B): 91B-98B.

9. O'Connell JB, Bristow MR. Economic impact of heart failure in The United States: time for a different approach. J Heart Lung Transplant 1994; 13: S107-12.

10. Parmely WW. Cost-effective cardiology: cost-effective management of heart failure. Clin Cardiol 1996; 19: 240-242.

11. Sinagra G, Di Lenarda A, Helede S, Rakar S, Sabbadini G, Davanzo M, Di Chiara C, Zanchi C, Carnie E. Epidemiologia clinica e gestione ambulatoriale dello scompenso cardiaco in base a criteri di costo/efficacia. G Riab 2000; XVI: 36-45.

12. Cleland JG, Swedberg K, Follath F, Komajda M, Cohen-Solal A, Aguilar JC, Dietz R, Gavazzi A, Hobbs R, Korewicki J, Madeira HC, Moiseyev VS, Preda I, van Gilst WH, Widimsky J, Freemantle N, Eastaugh J, Mason J; Study Group on Diagnosis of the Working Group on Heart Failure of the European Society of Cardiology. The Euro Heart Failure survey programme - a survey on the quality of care among patients with heart failure in Europe. Part 1: patient characteristics and diagnosis. Eur Heart J 2003 Mar; 24 (5): 442-63.

13. Opasich C, Tavazzi L. Di fronte alla nuova pandemia: quale organizzazione per la cura dei pazienti con insufficienza cardiaca? Cardiologia 1999; 554-565.

14. EARISA. Studio sulla epidemiologia e sull'assorbimento di risorse di ischemia, scompenso ed aritmia. G Ital Cardiol 1997; 27 (suppl. 2): $1-54$.

15. Komajda M, Follath F, Swedberg K, Cleland J, Aguilar JC, Cohen-Solal A, Dietz R, Gavazzi A, Van Gilst WH, Hobbs R, Korewicki J, Madeira HC, Moiseyev VS, Preda I, Widimsky J, Freemantle N, Eastaugh J, Mason J. Study Group on Diagnosis of the Working Group on Heart Failure of the European Society of Cardiology. The Euro Heart Failure Survey programme - a survey on the quality of care among patients with heart failure in Europe. Part 2: treatment. Eur Heart J 2003 Mar; 24 (5): 464-74.

16. Konstam M. Progress in heart failure management? Lesson from the real world. Circulation 2000; 102: 1076-1078.

17. Lega F. Orientare all'innovazione l'ospedale: aspetti strategici, organizzativi e gestionali.

18. Atti Convegno AIES Università Bocconi 4-5/11/2004 Milano.

19. Capomolla S, Pinna GD, et al. Heart failure case disease management program: a pilot study of home telemonitoring versus usual care. Eur Heart J 2004 F91-98. 


\section{TELECARDIOLOGIA: ASPETTI MEDICO-LEGALE}

\section{Massimo Cristina}

\section{Dipartimento di Medicina Legale, Azienda Ospedaliera di Busto Arsizio (VA)}

Se per telecardiologia si intende la possibilità di invio a distanza di informazioni relative al paziente cardiopatico per facilitarne il trattamento terapeutico ed assistenziale, significa pure che, se da un lato si spalancano nuovi orizzonti per un'assistenza continuativa e più efficace al paziente, dall'altro si inaugura un nuovo capitolo delle competenze e delle responsabilità dei vari operatori sanitari che non possono esulare da una puntualizzazione anche di carattere medico-legale.

In un'attività in cui le barriere degli spazi e dei tempi vengono superate dalle tecniche di telecomunicazione, il teatro delle competenze non si riduce più al semplice rapporto del paziente con il medico o con l'infermiere al suo capezzale, ma si amplia coinvolgendo nell'attività altre persone quali il medico di medicina generale, l'eventuale medico di altro ospedale, naturalmente il cardiologo del centro cardiologico di elevata specializzazione ed infine l'infermiere professionale, che viene ad assumere, sotto certi aspetti, il vero ruolo cardine di tutto il progetto terapeutico-assistenziale.

Si aprono a questo punto nuovi aspetti di competenza e, naturalmente, l'assunzione di nuove responsabilità in capo all'infermiere, la cui maggior autonomia operativa ed il cui ruolo di anello fondamentale in seno alle équipes è stato peraltro sempre meglio delineato dal graduale divenire della normativa speciale a partire dagli anni novanta.

Perché l'infermiere professionale possa disporre di questa autonomia operativa non è però sufficiente che sia in possesso di tutti i requisiti formali e sostanziali per esercitare la propria attività: occorre che nella fattispecie ottenga dall'avente diritto (il paziente) un consenso, generico e specifico ad operare, che è ciò che rende appunto lecito il suo agire.

Per consenso generico si intende l'autorizzazione del paziente ad essere inserito in un programma di telecardiologia e per consenso specifico l'autorizzazione per tutta l'attività dell'infermiere professionale in ordine alla rilevazione ed all'invio dei dati clinici, oltre che per le prestazioni terapeutiche ed assistenziali dirette.

Sarà quindi indispensabile che il modulo di consenso approntato per i servizi di telecardiologia contenga una breve ma dettagliata informazione sul significato e sull'utilità dell'inserimento del soggetto nel programma di telecardiologia ed inoltre (ciò che è fondamentale) l'autorizzazione del paziente anche per l'attività autonoma, domiciliare e non, dell'infermiere professionale nell'ambito di quanto stabilito dal programma di monitoraggio telematico.

In tale contesto l'infermiere dovrà agire con la maggior cautela possibile, non travalicando le proprie specifiche competenze; dovrà seguire norme di scrupolosa accuratezza nel raccogliere i dati clinici e nel trasferirli sotto forma di informazione al Centro Cardiologico di elevata specializzazione e dovrà attenersi rigorosamente alle disposizioni dello specialista cardiologo nell'esecuzione del trattamento terapeutico.

Solo con il più assoluto scrupolo operativo ed altrettanto rigore metodologico ad ogni livello della catena diagnostico-terapeutico-assistenziale è infatti ipotizzabile, anche sotto l'aspetto tecnico, la proficua realizzazione di un programma tanto innovativo, quanto, al contempo, ambizioso.

Si impone a questo punto un obbligo di puntualizzazione anche in riferimento alle competenze dei singoli operatori sanitari, che intervengono nell'attività di monitoraggio domiciliare del paziente, dacché, se la rilevazione dei dati è compito specifico dell'infermiere (talora persino del paziente), la formulazione del giudizio diagnostico e la scelta del trattamento terapeutico spettano strettamente al cardiologo; mentre l'esecuzione della terapia diviene una volta ancora competenza in larga misura dell'infermiere professionale, salvo i casi che impongano l'intervento diretto del cardiologo, ovvero l'ospedalizzazione del paziente.

È però in questa fase dell'attività che possono manifestarsi i rischi di possibile sconfinamento di ruolo dell'infermiere con possibilità di risvolti anche giudiziari, qualora dovessero verificarsi eventuali complicanze o eventi avversi come effetto di decisioni eccessivamente autonome, anche in ambito diagnostico-terapeutico.

$\mathrm{Da}$ ciò risulta facile comprendere come tutto l'agire dell'infermiere professionale pur supportato dal proprio sempre più vasto bagaglio di sapere teorico-pratico, debba in questo campo costantemente ispirarsi a principi di estrema prudenza, non dimenticando le norme di diligenza che i comuni rudimenti etici insegnano ed i numerosi casi di responsabilità professionale all'attenzione dell'Autorità Giudiziaria impongono.

Tuttavia i casi che vengono prospettati come espressione di reato o di illecito civile all'Autorità Giudiziaria stanno aumentando con intensità esponenziale. Pertanto è bene ricordare che, al verificarsi di un eventuale accidente con danno al paziente, l'infermiere può esser chiamato direttamente a rispondere per il danno causato dal proprio comportamento colposo (imperizia, imprudenza, negligenza o inosservanza di norme, regole, discipline...), mentre il cardiologo potrà rispondere ad identico titolo, vale a dire per responsabilità diretta conseguita dall'aver determinato un danno in prima persona al paziente per suo esclusivo errore colposo ovvero anche per responsabilità indiretta, a titolo di particolari aspetti di colpa, tra cui ci limitiamo a citare la culpa in eligendo, la culpa in committendo e la culpa in vigilando, venendo nel caso specifico a rispondere in solido con l'infermiere.

Va infine rammentato che di tutte le prestazioni effettuate nell'ambito del programma di telecardiologia è indispensabile conservare traccia documentale per ogni singolo paziente, esigibile e consultabile in ogni momento e per qualsiasi esigenza.

\section{LA GESTIONE DOMICILIARE DEL PAZIENTE POST-ACUTO: L'ESPERIENZA DEL POLICLINICO "UMBERTO I"}

\section{Francesco Fedele \\ Policlinico Umberto I, Roma}

Lo scompenso cardiaco rappresenta la patologia cardiovascolare più importante nei Paesi Occidentali per prevalenza, tasso di re-ospedalizzazione e i costi sanitari. ${ }^{1}$

La telecardiologia, negli ultimi anni, è stata impiegata con successo nella gestione di patologie cardiovascolari croniche, contribuendo al miglioramento dell'assistenza cardiologica, alla prevenzione delle reospedalizzazioni e alla riduzione dei costi gestionali. ${ }^{2}$

L'esperienza del Dipartimento di Malattie Cardiovascolari e Respiratorie del Policlinico Umberto I dell'Università "La Sapienza" di Roma in questo settore è iniziata circa 2 anni fa attraverso la partecipazione ad un progetto di telemedicina coinvolgente anche altre strutture ospedaliere cardiologiche romane, con l'obiettivo principale di valutare le reali potenzialità di un sistema domiciliare di telecardiologia, in termini di qualità della vita e tasso di re-ospedalizzazione, per pazienti con scompenso 
cardiaco cronico, nonché della possibilità di garantire l'aderenza al trattamento terapeutico per razionalizzare le risorse umane, economiche e logistiche.

A partire dal dicembre 2003 fino al settembre 2005, 138 pazienti con scompenso cardiaco cronico (III-IV classe funzionale NYHA) e disfunzione ventricolare sinistra $(\mathrm{FE}<40 \%)$ sono stati assegnati a due gruppi, dopo le dimissioni ospedaliere: uno seguito con telecardiologia domiciliare $(\mathrm{n}=73)$ ed uno con le cure convenzionali $(n=65)$.

In considerazione del rischio di questi pazienti di andare incontro a "morte cardiaca improvvisa" per eventi tachiaritmici e del fatto che tali eventi si manifestano in maggioranza proprio al domicilio del paziente stesso, ogni nucleo familiare, dopo adeguato addestramento alle manovre BLS-D di almeno uno dei componenti, veniva fornito di un defibrillatore esterno semiautomatico.

Il sistema di telecardiologia domiciliare si basa sul monitoraggio giornaliero di: elettrocardiogramma, pressione arteriosa non invasiva, saturimetria, bilancio idrico e peso corporeo, inviati dal domicilio del paziente direttamente alla postazione ospedaliera, attiva 24 ore su 24 , tramite una normale via telefonica, per un periodo di almeno tre mesi.

Vengono inoltre prese in considerazione sia la percentuale di re-ospedalizzazione sia la qualità di vita tramite la somministrazione del Minnesota Living With Heart Failure Questionnaire, all'inizio ed al termine del periodo di trattamento con il metodo di telecardiologia domiciliare.

Le caratteristiche della popolazione presa in esame, al momento del ricovero ospedaliero, è riassunto nella tabella 1 .

I risultati sino ad ora conseguiti hanno mostrato come il periodo di degenza ospedaliera medio nel gruppo di controllo era di 9,8 giorni contro gli 8,2 giorni rilevati per il gruppo "telecardiologia", mostrando una riduzione di 1,6 giorni $(16,3 \%)$, grazie alla possibilità offerta dal monitoraggio domiciliare di rilevare i principali parametri vitali ed eventuali condizioni precipitanti o alterazioni dello stato emodinamico, consentendo un precoce e rapido intervento da parte del medico.

Infatti gli interventi cui i medici risponditori sono stati più spesso chiamati sono quelli di drug tritration, cioè dell'ottimizzazione della terapia, in considerazione della variabilità delle condizioni di salute dei pazienti e della loro compliance, in particolare di quella con diuretici, beta-bloccanti ed ACE-inibitori, in accordo con i dati che stimano la mancata aderenza e/o compliance da parte del paziente al piano terapeutico come causa principale di reospedalizzazione e instabilizzazione. ${ }^{3}$

In una piccola percentuale $(12 \%)$ sono stati richiesti interventi in "urgenza", e solamente il 3\% ha richiesto l'ospedalizzazione.

La percentuale di reospedalizzazione risulta, poi, minore nel gruppo "telecardiologia" rispetto a quello di controllo (rispettivamente $3 \%$ vs. $15,5 \% ; p<0,05$ ).

Non si sono registrati nel periodo considerato eventi aritmici che hanno richiesto l'utilizzo del defibrillatore esterno semiautomatico.

In questo ambito, comunque, si sono registrati con soddisfazione ottimi risultati nell'addestramento dei conviventi dei pazienti alle manovre BLS ed all'utilizzo del defibrillatore in modalità semiautomatica, confermati dai re-training che hanno dimostrato una buona acquisizione delle nozioni di base.

Al termine del periodo di trattamento con il sistema di telecardiologia sono stati somministrati appositi questionari che hanno dimostrato un alto grado di soddisfazione e una maggior qualità nelle cure tra i pazienti del gruppo "telecardiologia"; si sono altresì dimostratati l'efficacia della telecardiologia ed il continuo contatto con il medico cardiologo come strumento di "protezione psicologica".
Tabella 1. - Caratteristiche della popolazione

\begin{tabular}{cccc}
\hline Classe NYHA & N. pazienti & Classe KILLIP & N. pazienti \\
\hline Classe I & 0 & Classe I & 0 \\
Classe II & 0 & Classe II & 11 \\
Classe III & 53 & Classe III & 58 \\
Classe IV & 20 & Classe IV & 4 \\
\hline
\end{tabular}

Questi dati dimostrano l'efficacia di un sistema di ospedalizzazione domiciliare basato su metodiche di telecardiologia, incrementando la qualità delle cure nel senso di una loro ottimizzazione, riducendo il numero delle reospedalizzazioni nei pazienti con scompenso cardiaco cronico e, di conseguenza, le spese gestionali.4,5

Tutto ciò pone le basi per un futuro ampliamento del progetto ed una diffusione di sistemi di "reti di ospedalizzazione domiciliare" al territorio regionale ed a quello nazionale, con l'obiettivo di stilare al più presto linee guida in materia di telemedicina, per la standardizzarne delle metodiche utilizzate e della raccolta dei risultati ottenuti nelle varie esperienze.

\section{Bibliografia}

1. Sharpe N, Doughty R. Epidemiology of heart failure and ventricular dysfunction. Lancet 1998; 352 (suppl. I): 3-7.

2. Wasson J, Gaudette C, Whaley F, Sauvigne A, Baribeau P, Welch $\mathrm{G}$. Telephone care as a substitute for routine clinic follow-up. JAMA 1992; 267: 1788-1793.

3. Casalino L, Gillies RR, Shortell SM, Schmittdiel JA, Bodenheimer T, Robinson JC, Rundall T, Oswald N, Schauffler H, Wang MC. External incentives, information technology, and organized processes to improve health care quality for patients with chronic diseases. JAMA 2003; 289: 434-441.

4. Grady KL, Dracup K, Kennedy G, et al. Team management of patients with heart failure. Circulation 2000; 102: 2443-2456.

5. Roglieri JL, Futterman R, McDonough KL, et al. Disease management interventions to improve outcomes in congestive heart failure. $\mathrm{Am}$ J Manag Care 1997; 3: 1831-1839.

\section{LA FORMAZIONE DELLE FIGURE SANITARIE: IL MEDICO}

\section{Amerigo Giordano}

Divisione di Cardiologia, IRCCS Fondazione Salvatore Maugeri, Istituto Scientifico di Gussago/Lumezzane (BS) E-mail:agiordano@fsm.it

La telemedicina, quale applicazione delle nuove tecnologie telematiche in campo sanitario, svolge oggi un ruolo fondamentale nell'interconnessione tra diversi utenti (piccoli ospedali periferici e strutture ospedaliere o universitarie di riferimento, paziente ed ospedale, medici del territorio, paziente e ospedale, etc...).

La telecardiologia, nell'ambito della telemedicina, ha assunto un ruolo di primo piano poiché le affezioni cardiovascolari sono tra le più rappresentate nella popolazione, soprattutto senile, e per esse è ampiamente dimostrata la bontà del rapporto costo-beneficio in termini di vite salvate e di recupero funzionale precoce. L'avvio di un servizio di telemedicina in una struttura ospedaliera è in genere caratterizzato dalla mancanza di risorse umane dedicate; di solito vi è una ridistribuzione di risorse provenienti da altri percorsi e dedicate alla telemedicina per un tempo parziale.

Occorre quindi: identificare e formare le risorse umane esistenti, creare nuove risorse umane proporzionali alla crescita del servizio, inserire il concetto di sviluppo e formazione delle risorse umane in tutte le specialità in cui si pensa di applicare la telemedicina.

Per quanto concerne la specifica attività di formazione che abbiamo istituito nel nostro Servizio di Telemedicina si sono stabiliti i seguenti criteri: 
- Gli specialisti in cardiologia hanno un'età compresa tra 35-60 anni e un'esperienza almeno quinquennale di Unità di Terapia Intensiva Coronarica.

- Verifica dei requisiti: di carattere professionale (laurea in medicina e chirurgia, specializzazione, esperienza in ambito lavorativo cardiologico); di carattere operativo (PC, ADSL o ISDN con numero telefonico aggiuntivo, conoscenza dei principali sistemi operativi informatici, FAX di emergenza).

- Condivisione delle principali linee guida delle patologie cardiache.

- Adozione e formazione sulle principali flow-chart operative concernenti i sintomi principali (cardiopalmo, dispnea etc...).

- Valutazione di almeno 50 casi clinici attraverso l'ausilio di CD-rom.

- Adozione e formazione sulle principali procedure di lavoro.

- Invio di dati trimestrali di aggiornamento (diagnosi di dimissione di tutti i pazienti inviati al Pronto Soccorso, segnalazione di pazienti che si sono recati ad un Pronto Soccorso dopo un teleconsulto che non lo richiedeva).

- Invio dei tracciati ECGgrafici in cui il referto non veniva ritenuto corretto (errori gravi; errori lievi).

- Tracciati elettrocardiografici: rilettura completa con segnalazione di errori gravi (erronea interpretazione del ritmo, disconoscimento di aritmie e/o blocchi etc...) ed errori lievi (non riconoscimento di deviazione assiale sx, etc...).

- Registrazione audio di tutte le chiamate telefoniche.

- Archiviazione di tutti i test eseguiti sulle chiamate (concordanza con le principali linee guida, etc...) ed analisi degli errori. Analisi di un periodo temporale di 8 mesi (Febbraio-Settembre 2004) di 10007 chiamate risposte da 26 cardiologi: Errori gravi: 45 (0.44\%) Errori lievi: $364(3.8 \%)$.

- Il personale medico è affiliato al servizio con una media di anni $5 \pm 2$ anni di lavoro ed una media di circa 300 chiamate/mese ricevute negli ultimi due anni.

- L'appropriatezza diagnostica del servizio sostenuto dal personale medico cardiologico è stata analizzata e pubblicata ed ha dato un risultato del $94 \%$.

Descrizione del flusso gestionale specifico:

- arrivo di una chiamata telefonica generata dal paziente

- riconoscimento automatico del chiamante, da parte del call center, tramite il numero di telefono memorizzato

- instradamento della telefonata al primo operatore tecnico libero

- apertura automatica della cartella clinica del chiamante su personal computer dell'operatore tecnico

- attivazione della procedura "nuova chiamata" che prevede l'immissione di dati specifici relativi alla chiamata (per controllo e/o sintomi)

- ricezione delle tracce elettrocardiografiche

- messa in comunicazione telefonica dell'utente con lo specialista o la nurse in servizio

- il cardiologo consulente, dalla sua postazione remota ospedaliera, in tempo reale ed in connessione col database centrale (tramite terminal-server), esamina la cartella clinica ed il tracciato inviato (confrontandolo con il basale eventualmente memorizzato)

- raccolta da parte dello specialista e della nurse di informazioni riguardanti anamnesi e sintomi

- teleconsulto medico specialistico

- refertazione on-line del tracciato elettrocardiografico

- chiusura del contatto telefonico.

Questa attività di formazione ci ha permesso di istituire un pool di "TeleCardiologi" affidabili ed efficienti. Questa metodica di formazione, sperimentata in cardiologia, è stata poi implementata nelle altre discipline.

\section{ASPETTI TECNOLOGICI ED ORGANIZZATIVI}

\author{
Fulvio Glisenti \\ Consorzio CO.ME.TE, Brescia
}

La Telecardiologia, intesa come strumento d'integrazione ospedale-territorio, è una pratica remota d'interscambio dati, immagini, testi attraverso un network di comunicazione la cui progettazione coinvolge due aspetti: quello tecnologico e quello organizzativo.

Per quanto attiene agli aspetti tecnologici dobbiamo fare riferimento a ulteriori quattro aspetti: infrastruttura di connessione, infrastruttura di accesso ai servizi, infrastruttura di sviluppo e gestione dei servizi, convergenza tecnologica.

Per infrastruttura di connessione pensiamo sostanzialmente alla banda larga cioè all'ambiente tecnologico (telecomunicazioni) che consente il trasporto di grandi quantità di dati in breve tempo. Alcune tecnologie trasmissive sono oggi ormai mature ed efficaci: ISDN, xDSL, fibra ottica, Wireless local loop (Wi-Fi), satellite. Tra queste xDSL (Digital Subscriber Loop) appare quella più diffusa e promettente dal punto di vista costo/efficacia; è un insieme di tecniche trasmissive (ADSL, SDSL, VDSL) che consentono di fornire servizi a banda larga in area d'utente utilizzando i normali doppini in rame delle linee telefoniche, già installati. Attualmente le versioni più diffuse in Italia prevedono, per l'utenza residenziale 640 $\mathrm{kbit} / \mathrm{s}$ verso l'utente e $128 \mathrm{kbit} / \mathrm{s}$ verso la rete mentre, per l'utenza "business", 2 Mbit/s verso l'utente e $512 \mathrm{kbit} / \mathrm{s}$ verso la rete. Altre tecnologie quali Powerline (tecnologia che consente la trasmissione di flussi numerici utilizzando le esistenti linee di distribuzione dell'energia elettrica) appaiono oggi ancora immature. Nell'ambito dell'infrastruttura di connessione, vi è poi la necessità della banda lunga, vale a dire di connessioni always-on, a un costo fisso contenuto, per l'home-care. Se passiamo ad analizzare l'infrastruttura di accesso, è fondamentale che i sistemi di telemedicina siano in grado di comunicare non solo con altri sistemi analoghi, al fine di garantire l'interoperabilità fra centri diversi, ma anche con tutti i sistemi informativi sanitari. L'adozione di standard $\mathrm{HW}+\mathrm{SW}$ è indispensabile e consente di risolvere questi problemi. Il contesto della telesanità ha inoltre una chiara necessità di strumenti che garantiscano la sicurezza intesa come sicurezza nell'accesso ai servizi, sicurezza delle reti, autenticazione attraverso la firma elettronica. Il rischio di violazione dei sistemi sempre in linea (in relazione alle connessioni always-on) è di rilevanza tale da aver motivato una specifica raccomandazione di intervento delle Autorità competenti (ricorso a VPN, crittografia).

L'infrastruttura di sviluppo e gestione dei servizi passa, a nostro avviso, attraverso gli ASP, Application Service Provider. L'ASP è un aspetto evoluto dell'outsourcing in cui $\mathrm{HW}+\mathrm{SW}$ e competenze professionali non sono localizzate nella sede degli utilizzatori ma risiedono nella sede del fornitore del servizio. La modalità di erogazione ASP è indispensabile per avvalersi di servizi di elevato livello qualitativo senza doversi necessariamente dotare di strumenti e competenze onerose. È infine auspicabile la convergenza su un'unica infrastruttura di servizi di fonia e dati (finora confinati in reti dedicate) che utilizzi open source, software a codice sorgente aperto (es. Linux) con formato aperto $\mathrm{Xml}$.

Per quanto riguarda gli aspetti organizzativi dell'integrazione ospedale-territorio questi passano attraverso l'infrastruttura umana che, analogamente all'infrastruttura tecnologica, è assai complessa. La scommessa della telemedicina è far lavorare insieme persone di diversa estrazione professionale in un ambiente tecnologico molto complesso e la tecnologia, che dovrebbe aiutare, spesso è ulteriore fonte di complicazioni, si rivela ad esem- 
pio"user-unfriendly" oppure soffre del "digital divide": divisione tra coloro che usano Internet e quelli che non lo fanno. Recenti studi evidenziano che il gruppo di coloro che non usano Internet non è omogeneo ma si divide tra coloro che sono interessati ad Internet, ma non sono in grado di connettersi per varie ragioni (economiche e di alfabetizzazione o perché discriminati in aree più o meno sviluppate del Paese, come ad esempio il Nord e il Sud) e in coloro che non hanno interesse a connettersi.

Immaginare l'integrazione ospedale-territorio è organizzare e dare un ruolo ai Centri Servizi, intesi come centrali operative di smistamento-chiamate e monitoraggioutenti. Un Centro Servizi dev'essere nel contempo un call-center (servizi principalmente in voce) e un contactcenter (web-services, fax, e-mail, video). Con piattaforme tecnologiche dedicate (hardware+software) deve fornire all'utente diverse tipologie di servizi, attivi in risposta $24 / 24$ ore per l'intero anno. Attraverso team di risorse umane "formate ed esperte", deve offrire servizi a seconda dei bisogni ed essere assimilabile ad ASP che, in modalità-outsourcing, gestiscono in tempo reale le esigenze (in voce, dati, immagini) di network di utenti. Un Centro Servizi deve poter offrire all'utenza teleconsulti/videoconsulti multispecialistici, second opinion, telenursing e triage infermieristico, refertazione di prestazioni strumentali (online, store and forward) tenendone traccia in un database protetto. Per rendere flessibile il sistema di risposta ed ottimizzare "on line" i tempi di interfaccia con l'utenza deve poter ricorrere al "tele-lavoro" che consente di remotizzare la parte professionale specialistica e di triage infermieristico.

Con questi intendimenti abbiamo realizzato un Centro Servizi di Telemedicina, attivo 24/24 ore, con una piattaforma tecnologica avanzata web-based (hardware+ software) articolata con database server, terminal server, web server, firewall, call center, workstation in rete LAN. Si è poi costruito un network tra ospedali, cliniche universitarie, medici di medicina generale, specialisti cardiologi e pazienti che permette di effettuare teleconsulti e refertazioni di tracce elettrocardiografiche "in tempo reale" anche per grandi volumi di traffico in contemporanea. Il processo attraverso il quale il Centro Servizi realizza la propria attività prevede la remotizzazione del teleconsulto professionale, con compilazione della cartella clinica informatizzata, attraverso un utilizzo spinto del telelavoro.

Sono operative due modalità di telelavoro: l'una tramite terminal server e l'altra web-based. Per entrambe le modalità specialisti cardiologi, addetti al teleconsulto, ed infermieri professionali, addetti al nursing-triage degli utenti, adottano un approccio di "telelavoro" così strutturato: linea telefonica domiciliare dedicata al servizio vocale di teleconsulto, personal computer connesso al sistema centrale tramite internet, connessione permanente in banda larga ISDN o "always on" ADSL.

La modalità terminal server si contraddistingue per l'utilizzo di un'interfaccia software di sicurezza-riservatezza (VPN) dati per controllare l'accesso internet in modo che il consulente accede direttamente, in chiaro, alla cartella clinica nel database centrale; il personal computer remoto funziona in emulazione terminale.

La modalità web-based prevede invece, in connessione al web server tramite doppia password d'accesso di lettura-scrittura, la visione e l'aggiornamento ogni due minuti della cartella clinica resa anonima, con il solo codice identificativo dell'utente.

La realizzazione del servizio avviene attraverso un flusso informativo che prevede: arrivo al call center di una chiamata telefonica (generata da pazienti, da medici, da strutture sanitarie, etc...); apertura automatica della cartella clinica del chiamante; attivazione della ricezione dell'elettrocardiogramma; messa in comunicazione telefonica dell'utente con lo specialista/nurse in servizio che dalla loro postazione domiciliare, in connessione in- ternet, visualizzano la cartella clinica e l'ECG, raccolgono informazioni riguardanti anamnesi e sintomi; a seguire attività di telenursing infermieristico con aggiornamento dati e teleconsulto specialistico; refertazione on line delle tracce ecgrafiche, invio all'utente via e-mail, fax, del tracciato refertato e firmato.

Il tempo medio di una prestazione in "telelavoro" (contatto con la centrale operativa, teleconsulto, refertazione, rinvio del referto via e-mail, fax o pubblicazione su web) è di 7-10 minuti.

In questo network il ruolo del personale infermieristico è andato definendosi in questi ultimi anni in letteratura mediante la messa a punto di programmi gestionali multidisciplinari; questi si propongono di fornire strumenti di follow-up che abbiano le basi in un network ricomprendente ospedale e territorio e facciano riferimento a procedure standardizzate/condivise da istituzioni ospedaliere e medici di medicina generale. In quest'ambito nasce una nuova figura di Infermiere "esperto nella materia" che possiede nuove e più ampie conoscenze tecnicoscientifiche quali capacità di: riconoscimento dei principali eventi aritmici ed ischemici rilevabili dalle tracce elettrocardiografiche inviate per via telefonica, oggettivazione della sintomatologia del paziente attraverso diagrammi di flusso preordinati, interazione telefonica, dal punto di vista umano, con il paziente, interazione professionale con il medico curante e il cardiologo del paziente, utilizzo degli strumenti informatici.

Le tecnologie attualmente utilizzate sono quelle più avanzate dell'ICT e consentono modalità di telelavoro in terminal-server (con VPN crittografate) o in web-server https (con dati anagrafici anonimizzati).

Gli sviluppi futuri sono indirizzati alla ricerca di standard per piattaforme ed applicativi condivisi (vedi progetto regione Lombardia CRS-SISS) in modo da garantire l'interoperabilità fra centri diversi e con i vari sistemi informativi sanitari. Dal punto di vista delle tecnologie trasmissive molto si sta facendo in ambito Wireless local loop (Wi-Fi), soprattutto con l'ultimo protocollo WiMax, che consente raggi d'azione di oltre $50 \mathrm{~km}$ e che verosimilmente andrà ad integrarsi con la nuova generazione di telefoni cellulari in "software defined radio", dotati di un microprocessore in grado, per ogni trasmissione, di scegliersi in quel momento il canale radio più conveniente con maggiore capacità trasmissiva (su rete fissa o mobile). L'ambiente più promettente è quello dei Web Services per la modalità ideale di sviluppo e gestione dei servizi (tecnologie standard consolidate, linguaggio XML, accessibilità, connettività multicanale, interoperabilità dei servizi in rete, protocolli di interazione).

Le tecnologie dell'ICT possono fare molto per la sanità ma è necessario sviluppare nel prossimo futuro l'intero sistema paese con una coerente visione strategicomultidisciplinare che, eliminando il "digital divide", sviluppi le necessarie competenze.

\section{LA FORMAZIONE DELLE FIGURE SANITARIE: L'INFERMIERE}

Giovanna Martinelli, Doriana Baratti, Lucia Marchina, Simonetta Scalvini, Amerigo Giordano

\section{Servizio di Telemedicina, IRCCS Fondazione Salvatore Maugeri, Istituto Scientifico di Gussago/Lumezzane (BS) E-mail:gmartinelli@e-htn.it}

La necessità di nuovi modelli gestionali nasce dal progressivo invecchiamento della popolazione, dalla riduzione della mortalità nella fase acuta delle malattie cardiache e quindi dall'aumento delle patologie croniche con successivo incremento dei costi per il SSN. La gestione del pa- 
ziente sul territorio attraverso la telemedicina sembra poter essere un metodo efficace ed efficiente per assistere il paziente con patologie croniche. All'interno di questo nuovo servizio, nascono nuove professionalità che devono essere organizzate e formate, quali la figura dell'infermiere risponditore (IR) di un servizio di telemedicina.

L'organizzazione del modello è così strutturata: 1) verifica dei requisiti professionali e di attitudine comunicativa; 2) raccolta dati personali (curriculum vitae); 3 ) formazione; 4) procedure e protocolli di lavoro; 5) controllo di qualità; 6) valutazione annuale.

La verifica dei requisiti comprende quelli di carattere professionale (diploma o laurea infermieristica, esperien$\mathrm{za}$ in ambito lavorativo cardiologico ed in particolare in che settore) e quelli di carattere operativo (possesso di PC, linea telefonica ADSL/ISDN, conoscenza di base dei principali sistemi operativi informatici, FAX di emergenza).

La formazione comprende: test di ingresso di elettrocardiografia: refertazione di 20 ECG monotraccia (valido se $>80 \%$ esatto); aspetti generali di elettrocardiografia clinica (da personalizzare sulla base dei risultati del test d'ingresso); condivisione delle principali linee guida delle patologie cardiache; consegna e spiegazione delle principali flow-chart operative concernenti i sintomi principali (cardiopalmo, dispnea, etc...); valutazione di almeno 50 casi clinici attraverso l'ausilio di CD-ROM didattico; consegna e spiegazione delle principali procedure di lavoro; test finale; corso di comunicazione; addestramento alla compilazione della cartella informatizzata; primo turno di lavoro con affiancamento dell'infermiere teacher.

Alla formazione iniziale segue una formazione continua, caratterizzata da: riunioni bimestrali di aggiornamento professionale; test periodico sull'argomento trattato nella riunione precedente; test di ECG monotraccia: 3 tracciati mensili da eseguire durante il turno di lavoro.

Durante il periodo lavorativo si effettuano poi i controlli di qualità che comprendono: registrazione di tutte le chiamate telefoniche; rilettura di tutti i tracciati ECG grafici da parte dell'infermiere teacher; archiviazione di tutti i test eseguiti ed analisi degli errori.

Attraverso questo servizio il personale infermieristico formato ha gestito 5257 chiamate di cui 1482 legate ad un servizio di diagnostica del cardiopalmo e 3775 legate ad un servizio di telesorveglianza per il paziente con scompenso cardiaco cronico. In entrambi i casi, all'atto dell'arruolamento del paziente, l'infermiere deve: consegnare l'apparecchiatura al paziente; istruire il paziente sull'utilizzo della stessa; scegliere la derivazione da registrare; registrare una monotraccia basale; convalida di ritorno della monotraccia registrata; compilazione della cartella informatizzata. Il paziente, in presenza di un sintomo o per un controllo precedentemente concordato, chiama il centro servizi ed invia il tracciato. L'Infermiere Risponditore (IR) di turno visualizza la traccia, la confronta con quella basale e trascrive i sintomi. Se il tracciato ricevuto appare invariato rispetto al basale, l'IR lo archivia; in presenza di modifiche del tracciato o di dubbi rispetto alla sintomatologia del paziente, l'IR si metterà in contatto con il cardiologo di turno presso il Centro Servizi e chiederà un consulto on-line; chiusura del contatto telefonico.

Su 1482 chiamate dedicate al servizio diagnostico, l'IR ha richiesto la consulenza del cardiologo in 128 $(8.6 \%)$, mentre su 3775 chiamate del servizio di telesorveglianza, l'IR ha richiesto la consulenza del cardiologo in $386(10.2 \%)$.

\section{Conclusioni}

L'introduzione di nuove tecnologie può essere facilmente integrata nel lavoro svolto dagli infermieri per la gestione del paziente a condizione che si realizzino programmi di formazione ad hoc ed opportuni controlli di qualità sul lavoro svolto.

\section{TELECARDIOLOGIA E RETE EMERGENZA/URGENZA}

Maurizio Marzegalli

UO di Cardiologia, Azienda Ospedaliera San Carlo, Milano - Gruppo di Lavoro Emergenza cardiologica - ACEU1 Milano

\section{Introduzione}

Nella sindrome coronarica acuta, la riduzione del "ritardo evitabile" cioè del tempo perso prima che il paziente possa ricevere le cure adeguate, è una meta prioritaria. Per poter quindi intervenire tempestivamente, occorre una rete di intervento che inizi il triage del paziente sul territorio mediante l'esecuzione di un ECG 12 derivazioni (ECG 12d) e la trasmissione di questo ai reparti di Cardiologia.

\section{Telecardiologia in emergenza}

Per poter selezionare i pazienti che necessitano di un intervento di rivascolarizzazione non farmacologico, è necessario conoscere l'età, l'ECG 12d (per valutare se e in quante derivazioni è sopraslivellato il tratto ST), la frequenza cardiaca, la pressione arteriosa, lo stato di compenso del circolo rilevabile clinicamente e la saturazione arteriosa. Tutti questi dati oggi possono essere rilevati sul territorio e trasmessi nelle sedi opportune.

Numerose sono le soluzioni operative adottate nel mondo per realizzare le reti tra il territorio e le UCC. La letteratura è ricca di segnalazioni di come l'arrivo in ospedale con mezzi di emergenza e l'esecuzione di un ECG12d preospedaliero, specie se comunicato all'ospedale di destinazione, consenta di ridurre i tempi per l'inizio della terapia. Già nel ' 94 nel "National Heart Attack Alert Program" si affermava che "è stato ben dimostrato che il paziente con attacco cardiaco riceve un trattamento più rapido nel dipartimento di emergenza quando paramedici eseguono un ECG12d sul campo e lo trasmettono al dipartimento d'emergenza da un telefono cellulare. Il sistema di emergenza deve valutare la possibilità di eseguire un ECG12d a tutti i pazienti con sintomi e segni di attacco cardiaco. Idealmente tutti i veicoli dovrebbero avere la capacità di trasmettere un ECG12d da un telefono cellulare". ${ }^{1}$ Negli ultimi anni si è infatti dimostrato che è possibile eseguire sul territorio un ECG di qualità diagnostica e di trasmetterlo con successo in circa 2-4 minuti nell' $85 \%$ dei casi e che la specificità della diagnosi di infarto miocardico è passata così dal 68 al $95 \%$ ed il valore predittivo positivo dal $33 \%$ al $71 \% .^{2}$ Grazie alla esecuzione e trasmissione dell'ECG dal territorio, la riduzione del tempo intraospedaliero dall'arrivo al trattamento si è ridotto secondo i vari studi da 20 a 55 minuti.

In un altro studio nell'ambito del "Cincinnati Heart Project"3 si è dimostrata l'influenza dei mezzi di trasporto sul tempo medio del ritardo intraospedaliero: 64 minuti per i pazienti giunti con mezzi propri, 55 minuti se giunti con autoambulanze locali, 50 minuti per pazienti trasportati dopo aver eseguito un ECG12d non trasmesso all'ospedale, 30 minuti per i pazienti trasportati dopo aver eseguito un ECG12d ed averlo trasmesso all'ospedale ricevente.

Nell' ambito del "National Registry of Myocardial Infarction - 2 data base" 4 si è notato che la differenza tra $i$ pazienti che erano stati sottoposti ad ECG preospedaliero e quelli che non lo erano, benché il tempo di arrivo in ospedale fosse più lungo, era che i primi sono stati sottoposti più rapidamente e frequentemente a fibrinolisi, PTCA o by-pass e la loro mortalità intraospedaliera è risultata dell' $8 \%$ contro il $12 \%(\mathrm{p}<0.001)$.

Tra le raccomandazioni in caso di sindrome coronarica acuta espressi dalla "Task force 2: prehospital issues"

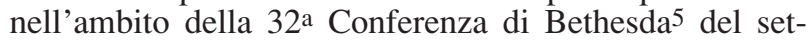
tembre 1999 spiccano le seguenti: 
a) Un programma di ECG12d preospedaliero deve essere inserito in un sistema di emergenza paramedico urbano e suburbano.

b) L'ECG12d preospedaliero deve essere comunicato al medico del dipartimento di emergenza ricevente prima dell'arrivo del paziente.

c) Pazienti con infarto miocardico e compromissione emodinamica, shock cardiogeno o altri elementi di alto rischio devono essere sottoposti ad un triage che li invii a centri dove sia possibile una rivascolarizzazione d'urgenza qualora il trasporto non superi i 30 minuti. Il triage deve essere eseguito il più presto possibile, preferibilmente sul campo o nel più vicino dipartimento di emergenza, a seconda dell'organizzazione locale.

Le Linee Guida internazionali6 (Guidelines 2000 for Cardiopulmonary Resuscitation and Emergency Cardiovascular Care. International Consensus on Scienze) autorevolmente ed esaurientemente indicano la strada da seguire per le emergenze cardiologiche; esse propongono come soluzione di Classe I la trasmissione dell'ECG 12d da sistemi di emergenza territoriale ai dipartimenti di emergenza. Le recenti linee guida per 1'IMASTE7 prevedono $^{2}$ il triage preospedaliero e l'utilizzo della telecardiologia per la scelta della terapia ottimale per il paziente e l'eventuale terapia fibrinolitica preospedaliera.

Anche in tutti i recenti progetti di RETE proposti dall'ANMCO per l'emergenza è auspicato l'utilizzo della telecardiologia per la trasmissione dei dati

Per le emergenze cardiologiche quindi si va sempre più verso una metodica di intervento e stabilizzazione in sede, nota come "stay and stabilize" che deve essere messa in atto il più precocemente e rapidamente possibile. In particolare l'esecuzione di un ECG $12 \mathrm{~d}$ è un intervento che richiede solo pochi minuti ma che cambiano il corso degli eventi.

\section{Struttura della Rete della Città di Milano}

Il Gruppo di Lavoro per l'Emergenza Cardiologia Extraospedaliera attualmente opera nell'Area di Coordinamento della Emergenza Ugenza (ACEU 1), ed ha rivolto la sua attenzione innanzitutto alla fase preospedaliera. Per affrontare le SCA e in particolare lo STEMI, il progetto ha previsto all'inizio di dotare l'elicottero e i 5 MSA di monitor defibrillatore con ECG 12d con lettura automatica (LIFEPAK 12 EMS Medtronic). Sono state successivamente aumentate di numero le automediche che sono state dislocate anche sul territorio provinciale: attualmente sono 9. Successivamente è stata installata una rete telematica di comunicazione tra i mezzi MSA sul territorio e la CO 118 e tra queste e le Cardiologie - UTIC che ha iniziato la sua attività-nel Febbraio 2001. Si è proceduto quindi all'installazione presso la CO 118 e presso gli Ospedali sedi di DEA e di UTIC di centraline (Lifenet RS) di ricezione dei dati trasmessi dai MSA. Queste centra- line sono in grado di ricevere tutti i dati clinici di monitoraggio, l'ECG e una traccia di monitoraggio per analisi di particolari aritmie intercorse. Attualmente la trasmissione avviene per via telefonica secondo il sistema GSM. Le centraline sono connesse tra di loro mediante normale linea telefonica. La ricezione del solo ECG in una UTIC è possibile anche mediante un comune fax.

Grazie a questa rete è anche possibile iniziare una terapia specifica già sul territorio. E stato da poco approvato un nuovo protocollo operativo, che prevede, secondo le più recenti linee guida, l'utilizzo anche dei $\beta$-bloccanti, l'eparina e della terapia fibrinolitica preospedaliera, qualora sia possibile praticarla in assenza di controindicazioni assolute, entro un'ora dall'inizio dei sintomi e non vi sia a disposizione una struttura cardiologica in grado di eseguire immediatamente una PCI primaria.

\section{Teletrasmissione dell'ECG a 12 derivazioni da parte} dei Mezzi di Soccorso Avanzato

I dati riportati si fondano sull'analisi degli ECG teletrasmessi dai MSA e ricevuti dalla CO 118. Dal 1 Feb-

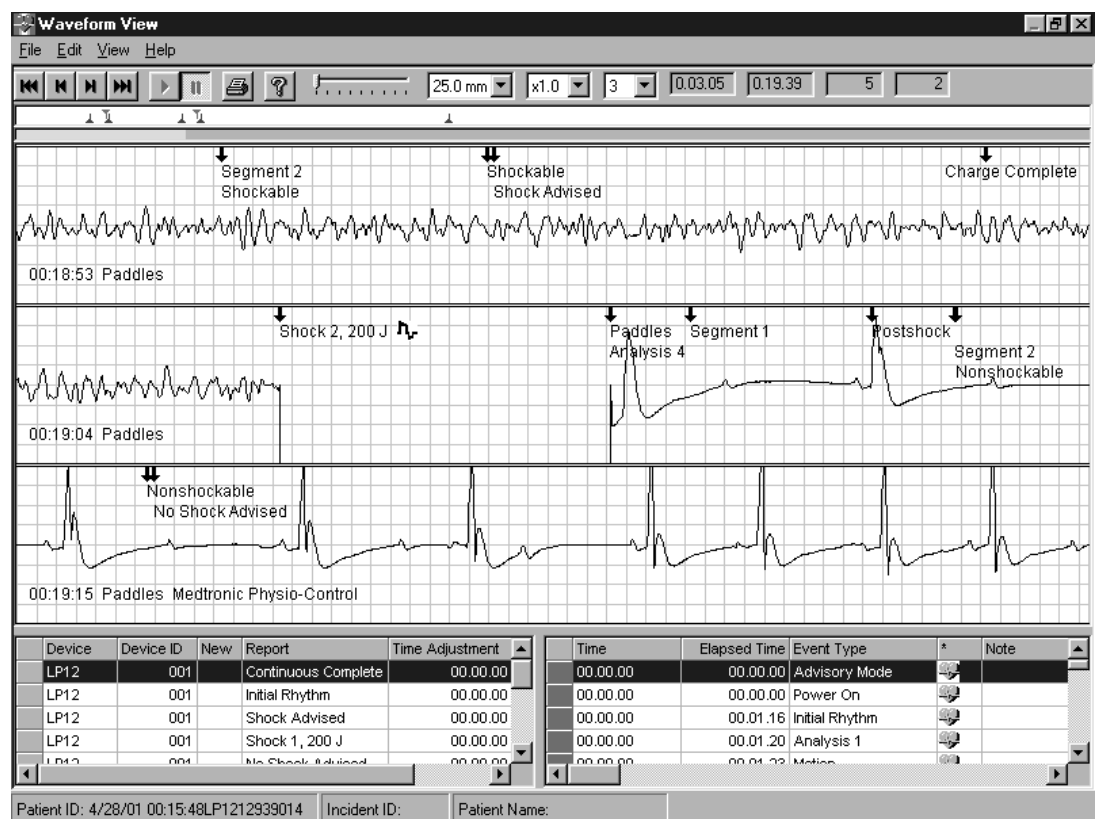

Figura 1. - Dati trasmessi da una ambulanza ALS di un soggetto con FV in corso di IMA.



Patient ID: 4/28/01 00:15:48LP1212939014 $\mid$ Incident ID: |Patient Name:

Figura 2 - Tracciato ECG 12d trasmesso nello stesso caso pochi minuti dopo la defibrillazione. 
braio 2001 al 1 Maggio 2005 sono stati soccorsi dai MSA con trasmissione dell'ECG 6.821 pazienti con sospetto attacco cardiaco, con una media di 177 pazienti/mese e un trend in ascesa (21 pazienti nel primo mese, 198 pazienti nel Dicembre 2004).

Il tracciato ECG è stato eseguito, in base ai tempi registrati automaticamente dal Lifepak 12, a una media di $5 \pm 4$ minuti dopo l'arrivo del MSA. Il tracciato è stato ricevuto a una media di $5 \pm 3$ minuti dopo la sua esecuzione. La trasmissione è riuscita al primo tentativo nell' $86 \%$ dei casi.

Tra i casi con ECG trasmessi in CO 118 in 4 anni sono state documentate 46 fibrillazioni ventricolari (figura 1) che sono state prontamente cardiovertite con un numero totale di $131 \mathrm{DC}$ shock. In questi casi l'aritmia era insorta durante il trasporto o mentre il paziente attendeva l'arrivo dei soccorsi chiamati per dolore toracico sospetto per angina. L'ECG eseguito dopo la ripresa del ritmo spontaneo e trasmesso alla $\mathrm{CO} 118$ ha documentato in 24 casi la presenza di sospetto STEMI (figura 2). In alcuni casi si trattava di un'onda di lesione in sede inferiore di estensione limitata. Anche in questi casi, data la instabilità elettrica si è provveduto, ove possibile, ad un triage primario verso un centro per PCI primaria. Questi dati sono analizzati all'interno del progetto di defibrillazione precoce denominato MILEDY (MILano Early DefIbrillation).

Si sono documentati frequentemente fenomeni transitori regrediti prima dell'arrivo in Pronto Soccorso come onde di lesione, blocchi atrioventricolari, tachicardie ventricolari. Per questo motivo è stata inserita nel protocollo l'indicazione a eseguire più tracciati durante il soccorso preospedaliero, specialmente al variare della sintomatologia. Nell'ultimo anno sono stati eseguiti un unico tracciato ECG nel 58\% dei casi, 2 nel 28\%, 3 nel 9\%, 4 nel 3\%, fino a un massimo di 8 in un caso isolato.

In $686(20 \%)$ dei 3427 tracciati trasmessi che sono stati successivamente rivisti da un cardiologo presso la CO 118, la diagnosi ECG è risultata di sospetto IMA: in particolare in $381(11.1 \%)$ di sospetto STEMI e in 305 (8.9\%) di sospetta sindrome coronarica acuta senza ST sopraslivellato (NSTEMI). 816 ECG $(23.9 \%)$ sono risultati normali, $1926(56.1 \%)$ presentavano anomalie del ritmo o della ripolarizzazione senza caratteristiche di emergenza (fibrillazione atriale in 137, pari al $4 \%$ dei tracciati). L'interpretazione automatica dei tracciati è risultata corretta nell' $80 \%$ dei casi. Nell' $11 \%$ dei casi il tracciato non è stato letto per presenza di artefatti (tremori, elettrodi staccati, artefatti grossolani), nel $9 \%$ dei casi l'interpretazione automatica è risultata errata per disturbi del ritmo, della conduzione o della ripolarizzazione senza tuttavia conseguenze cliniche sulla situazione di emergenza. La diagnosi di "IMA ACUTO", che compare come diagnosi automatica, è risultata errata nell' $1,1 \%$ dei casi; in particolare i falsi positivi sono stati $0,3 \%$ e i falsi negativi $0,8 \%$. Eseguendo più tracciati successivi, in caso di falsa negatività per minimo incremento del tratto $\mathrm{ST}$, la lettura è risultata corretta con l'evolversi della situazione clinica.

\section{Conclusioni}

Le emergenze cardiologiche necessitano di apparecchiature particolari, di una organizzazione specifica e di "reti" operative telematiche che colleghino tutti gli anelli della catena di intervento.

La tecnologia, come abbiamo visto, offre oggi soluzioni adeguate ad affrontare meglio questi problemi sia con sistemi di monitoraggio e di intervento terapeutico che con sistemi di trasmissione dati. Tuttavia la tecnologia da sola non basta. Occorrono protocolli condivisi tra tutti gli operatori in gioco.

\section{Bibliografia}

1. National Heart Attack Alert Program. National Heart, Lung and Blood Institute Action Alert, Staffing and Equipping Emergency Medical Services Systems: Rapid Identification and Treatment of Acute Myocardial Infarction. NIH Publication No.93-3304.

2. Aufdereide TP, Hendley GE, Woo J, Lawrence S, Valley V, Teichman SL. A prospective evaluation of prehospital 12 - lead ECG application in chest pain patients. J Electrocardiol 1992; 24; Suppl: $8-13$.

3. Kereiakes DJ, Gibler WB, Martin LH, Pieper KS, Anderson LC Relative importance of emergency medical system transport and the prehospital electrocardiogram on reducing hospital time delay to therapy for acute myocardial infarction: a preliminary report from the Cincinnati Heart Project. Am Heart J 1992; 123: 835-40.

4. Canto J, Rogers W, Bowlby L, French W, Pearce D, Weaver D, for the National Registry of Myocardial Infarction 2 Investigators. The Prehospital Electrocardiogram in Acute Myocardial Infarction: it is full potential being realized. J Am Coll Cardiol 1997; 29: 498-505.

5. Hutter AH, Weaver WD, Task Force 2. Acute acoronary Syndromes: Section 2A - Prehospital Issues. J Am Coll Cardiol 2000; 35: $825-880$

6. Guidelines 2000 for Cardiopulmonary Resuscitation and Emergency Cardiovascular Care. International Consensus on Scienze. Circulation 102, 8, August 22, 2000.

7. ACC/AHA Guidelines for the management of patients with ST elevation Myocardial infarction Circulation 2004; 110.

\section{RIABILITAZIONE E MODELLI DI GESTIONE DOMICILIARE DEL PAZIENTE CON SCOMPENSO CARDIACO CRONICO: RISULTATI PRELIMINARI DELLO STUDIO HHH}

Andrea Mortara*, Gian Domenico Pinna ${ }^{\circ}$,

Soccorso Capomolla ${ }^{\circ}$, Roberto Maestri ${ }^{\circ}$, Paul Johnson§, Maria Teresa La Rovere ${ }^{\circ}$, Piotr Ponikowski ${ }^{\circ}$,

Luigi Tavazzi**, Peter Sleight\$, a nome dei ricercatori dello Studio $\mathrm{HHH}$

* Divisione di Cardiologia e Unità Scompenso Cardiaco, Policlinico di Monza, Monza

- Divisioni di Bioingegneria e di Cardiologia, IRCCS Fondazione Salvatore Maugeri, Istituto Scientifico di Montescano $(P V)$

$\S$ University of Oxford, Departments of Cardiovascular Medicine and Telemonitoring Research Center, Nuffield Department of Obstetrics, John Radcliffe Hospital, Oxford, UK

๑ Department of Cardiology, Clinical Military Hospital, Wroclaw, Poland

** Divisione di Cardiologia, IRCCS, Policlinico S. Matteo, Pavia, Italy

\section{Introduzione}

Un numero crescente di pazienti affetti da scompenso cardiaco (SC) particolarmente nell'età avanzata, e l'esponenziale aumento della spesa sanitaria per questo tipo di patologia, impongono la necessità di ridisegnare i percorsi sanitari di cura.

Diverse esperienze di gestione dello $\mathrm{SC}$ in tutto il mondo e più recentemente anche in Italia, ${ }^{1-4}$ si sono basate su nuove modalità di assistenza integrata e di tipo multidisciplinare con la presenza sul territorio di personale medico e non-medico e con il maggiore o minore uso di nuove modalità di comunicazione. Lo sviluppo tecnologico ha reso disponibili nuovi sistemi di controllo a distanza delle condizioni cliniche del paziente affetto da SC attraverso sistemi di telemedicina. Tuttavia il possibile incremento dei costi, la compliance dei pazienti e la non certezza dei risultati clinici rende necessario che vengano condotti attenti studi randomizzati. 
Lo studio multicentrico HHH (Home or Hospital in Heart Failure) è stato disegnato per valutare un nuovo sistema di monitoraggio telemetrico domiciliare in pazienti affetti da SC al di fuori dall'ospedale. Il sistema di telemonitoraggio prevedeva il controllo e la trasmissione a distanza direttamente da casa dei pazienti, di alcuni parametri clinici e la registrazione Holter 24h, sempre domiciliare della attività cardio-respiratoria. Il progetto $\mathrm{HHH}$ integra una nuova infrastruttura gestionale del paziente con SC e una rigorosa valutazione clinica con importanti elementi di autogestione e controllo della malattia.

Nei modelli tradizionali di cura dello SC la misura dei più semplici parametri vitali è in genere eseguita dai medici o dal personale infermieristico dedicato sia in ospedale che a domicilio dei pazienti. Lo studio HHH sottolinea l'importanza di rilevare questi parametri vitali per mantenere la stabilità clinica, ma estende tale concetto alla possibilità di eseguire direttamente la misurazione con un sistema transtelefonico senza che avvenga lo spostamento di nessuna figura professionale. Inoltre viene testato un sistema per registrazioni di lunga durata dell'attività cardiorespiratoria che il paziente può auogestire a casa dopo una adeguata fase di training. Oggetto di studio è stata anche la valutazione della affidabilità e della utilità di questi sistemi di misurazione con una adeguata analisi costo-beneficio.

Non esistono attualmente studi che abbiano considerato la trasmissione transtelefonica di registrazioni holter $24 \mathrm{~h}$ della attività respiratoria. Nei pazienti con SC è stata infatti dimostrata un'alta prevalenza di disturbi dell'attività respiratoria (circa nel 50\% dei pazienti) che si manifestano con periodiche fluttuazione del volume respiratorio (respiro periodico) fino alla comparsa di alternanza di fasi di apnea di lunghezza variabile da 10-30 secondi e iperpnea (apnee centrali e respiro periodico di CheyneStokes). 5 Queste alterazioni centrali della dinamica respiratoria che si alternano spesso anche alla presenza di apnee ostruttive (OSA), si manifestano prevalentemente nelle ore notturne e determinano marcate oscillazioni della saturazione di $\mathrm{O}_{2}$ con incremento della attività simpatica e deterioramento emodinamico. $5-8$

Esistono pochi dati in letteratura sulla prevalenza di queste anomalie del pattern respiratorio soprattutto per periodi lunghi di osservazione (cioè più di una notte $\mathrm{o}$ un giorno) e non è noto se esse esercitino un fattore di instabilizzazione e peggioramento dei sintomi, favorendo più frequenti fasi di instabilizzazione. È stato suggerito come la presenza di severe apnee centrali possa identificare un gruppo di soggetti a maggior rischio di eventi e che il trattamento con la ventilazione tipo C-PAP possa ridurre la mortalità e la necessità di trapianto cardiaco a due anni solo in pazienti con alterazioni della dinamica respiratoria ma non in coloro che non le presentavano. ${ }^{9}$ In quest'ottica, lo studio HHH si pone l'obiettivo di determinare la prevalenza, la persistenza nel tempo e le correlazioni con possibili fasi di instabilizzazione emodinamica ed eventi di follow-up, delle alterazioni del pattern respiratorio soprattutto nelle sue forme più severe con apnee centrali importanti.

Inoltre dalla registrazione Holter $24 \mathrm{~h}$ della frequenza cardiaca sarà possibile determinare la variabilità della frequenza cardiaca che rappresenta una tecnica noninvasiva per la misura della attività simpato-vagale diretta al cuore. 10-11 Una bassa variabilità della frequenza cardiaca soprattutto dopo analisi spettrale nella componente LF è stato recentemente dimostrato essere un buon marker prognostico contribuendo alla stratificazione di pazienti con SC ad elevato rischio di morte improvvisa. ${ }^{12}$

\section{Obiettivi}

1) Confrontare differenti strategie di monitoraggio telefonico in pazienti con SC ad elevato rischio di instabiliz- zazioni con lo scopo di ridurre i costi dell'assistenza e migliorare la qualità di vita.

2) Valutare un nuovo sistema di trasmissione transtelefonica di ECG e attività respiratoria ottenute da registrazioni Holter $24 \mathrm{~h}$ eseguite direttamente a domicilio dal paziente

3) Definire la prevalenza, l'impatto clinico e il ruolo prognostico delle anomalie del pattern respiratorio, delle aritmie e delle alterazioni della variabilità della frequenza cardiaca sia in condizioni basali che durante le fasi di instabilità.

\section{Disegno e protocollo}

Lo studio HHH è disegnato come uno studio europeo di tipo multicentrico, randomizzato e controllato che è stato condotto con il supporto della Comunità Europea (Action line 10.1 "Public Health, proposal ${ }^{\circ}$ QLGA-CT2001-02424). Il trial ha coinvolto 3 nazioni (Regno Unito, Italia e Polonia) con un arruolamento previsto di 450 pazienti affetti da SC in due bracci:

- trattamento convenzionale $(\mathrm{n}=150)$

- monitoraggio clinico domiciliare $(n=300)$ questi ultimi randomizzati in tre gruppi secondo tre predefinite strategie di monitoraggio:

- gruppo 1) semplice contatto vocale per via telefonica con a disposizione anche una segreteria telefonica $24 \mathrm{~h}$ più terapia convenzionale;

- gruppo 2) come il gruppo 1, più la trasmissione settimanale dei parametri vitali (peso, frequenza cardiaca, pressione sistolica, score di fatica e dispnea, presenza di edema periferico);

- gruppo 3) come il gruppo 1 , più la registrazione $24 \mathrm{~h}$ una volta al mese di ECG, respiro e attività fisica.

In tutti i pazienti sia del gruppo controllo sia monitoraggio è stata eseguita la registrazione Holter pre-dimissione, mentre nei pazienti del gruppo 2, è stata anche eseguita la registrazione Holter mensile non utilizzata per il controllo clinico ma solo per scopi di ricerca.

L'arruolamento è durato 18 mesi con 12 mesi di follow-up per tutti i pazienti. Come end-point primario è stato considerato il numero di giornate di degenza per SC in qualsiasi reparto escluso le lungo-degenze (DRG 127). Sono stati poi considerati degli end-point secondari predefiniti che includono: a) giornate di degenza per qualsiasi causa e per causa cardiaca, b) evento composito (morte, ospedalizzazioni, e QoL), c) morte (cardiaca e non cardiaca), d) variazioni e compliance ai cambiamenti di terapia, e) valutazione dei costi ed efficacia degli interventi, f) prevalenza delle alterazioni del pattern respiratorio e di una depressa variabilità della frequenza cardiaca, g) ruolo di HRV, apnee centrali e fibrillazione atriale parossistica nell'insorgenza di fasi di instabilizzazione della malattia

\section{Sistema di telemonitoraggio}

Il sistema di monitoraggio comprendeva: 1) una segreteria telefonica attiva $24 \mathrm{~h}$ e una linea telefonica convenzionale sempre disponibile fra il personale infermieristico e il paziente, 2) un risponditore automatico interattivo (IVR, Ditta Apple Electronica srl, Torino) in grado di gestire la trasmissione periodica dei parametri vitali da casa del paziente all'ospedale, 3 ) un registratore $24 \mathrm{~h}$ (Ditta Marazza, Monza) gestibile autonomamente dal paziente a casa, in grado di registrare ECG, respiro e attività fisica, e di trasmettere in modo automatico le registrazioni dei segnali all'ospedale di riferimento.

Il protocollo si è avvalso dell'importante supporto tecnico della Bioingegneria della Fondazione Salvatore Maugeri di Montescano che ha messo a punto i seguenti software: a) per la gestione dei data base centrali e periferici, b) per la gestione della segreteria telefonica e delle telefonate in entrata e uscita dal centro arruolatore, c) per la raccolta, controllo qualità e rappresentazione grafica 
dei parametri vitali ed esami di laboratorio, d) per la gestione Holter 24h, e) per la gestione delle registrazioni Holter in arrivo e controllo qualità, f) per la lettura delle registrazioni di ECG, attività respiratoria e movimento, $\mathrm{g}$ ) per l'analisi della variabilità della FC e della attività respiratoria con produzione dei report per i Centri.

\section{Risultati}

L'arruolamento dei pazienti è stato completato nel giugno 2004 e il termine del follow-up di 12 mesi è avvenuto nel luglio 2005. Nelle tre nazioni sono stati arruolati 464 pazienti: $46 \%$ in Italia, $13 \%$ in UK, and $41 \%$ in Polonia. Tali pazienti sono stati randomizzati (1:2) con successo a terapia convenzionale $(n=157)$ e a monitoraggio domiciliare $(n=307)$ nelle tre pre-definite strategie: a) semplice contatto telefonico $(n=106)$, b) come la precedente più monitoraggio dei parametri vitali $(\mathrm{n}=98), 3)$ come la strategia 2 più monitoraggio periodico $24 \mathrm{~h}$ di $\mathrm{ECG}$, respiro e attività fisica $(n=103)$.

I dati preliminari sul telemonitoraggio (167 / 201 pazienti, 83\%) mostrano che vi è stata una corretta e ottimale trasmissione dei parametri vitali nel $71 \%, 82 \%$ e $60 \%$ rispettivamente dei pazienti in Italia, UK, e Polonia, con un totale di 38116 parametri trasmessi, e 551 messaggi vocali. Nelle tre nazioni partecipanti, i pazienti arruolati nella strategia 2 e 3 hanno registrato e trasmesso $i$ dati dell'Holter $24 \mathrm{~h}$ nel $87 \%$ (Italia), 70\% (UK) and 55\% (Polonia) dei casi, con un totale di 1954 registrazioni Holter da casa (analizzati 196 di 201 pazienti, 97.5\%). Ad una prima analisi, i dati suggeriscono una prevalenza di disturbi del respiro del 58\%, $42 \%$ e $55 \%$ rispettivamente nei tre paesi. Queste severe anomalie del pattern respiratorio sembrano persistere nel corso del follow-up in più del $60 \%$ dei pazienti.

È attualmente in corso l'analisi principale dello studio sugli effetti delle differenti strategie di monitoraggio per quanto riguarda gli end-point primario e secondari.

\section{Conclusione}

I dati iniziali dello studio HHH mostrano come, nei pazienti con SC cronico, il sistema di telemonitoraggio dei parametri vitali e della frequenza cardiaca e respiratoria nelle 24-ore sia fattibile, accurato e con un'alta compliance da parte dei pazienti. Se i dati clinici finali dello studio mostreranno una riduzione delle ospedalizzazioni e un miglioramento della qualità della vita tale sistema potrà contribuire ad una gestione più efficace dei pazienti con SC.

\section{Bibliografia}

1. McAlister FA, Stewart S, Ferrua S, McMurray JJJV. Multidisciplinary strategies for the management of heart failure patients at high risk for admission: A systematic review of randomized trials. $J \mathrm{Am}$ Coll Cardiol 2004; 44: 810-8.

2. Whellan DJ, Hasselblad V, Peterson E, O'Connor CM, Schulman KA. Metaanalysis and review of heart failure disease management randomized controlled clinical trials. Am Heart J 2005; 149: 722729.

3. Christopher O. Phillips. Comprehensive discharge planning with postdischarge support for older patients with congestive heart failure. JAMA 2004; 291: 1358-1367.

4. Capomolla S, Alunni G. La prevenzione delle recidive, modelli manageriali. In: "La prevenzione nello scompenso Cardiaco" 2005: 183-212.

5. Bradley TD, Floras JS. Sleep apnea and heart failure: Part II: central sleep apnea. Circulation 2003; 107: 1822-6.

6. Mortara A, Sleight P, Pinna GD, Maestri R, et al. Abnormal awake respiratory patterns are common in chronic heart failure and may prevent evaluation of autonomic tone by measures of heart rate variability. Circulation 1997; 96: 246-252.

7. Ponikowski P, Anker S D, Chua TP, Francis D, Banasiak W, Poole-Wilson PA, Coats AJS and Piepoli M. Oscillatory breathing patterns during wakefulness in patients with chronic heart failure. Clinical implications and role of augmented peripheral chemosensitivity. Circulation 1999; 100: 2418-2424.

8. Lanfranchi P, Braghiroli A, Bosimini E, et al. Prognostic Value of Nocturnal Cheyne-Stokes Respiration in Chronic Heart Failure. Circulation 1999; 11: 1435-1440.
9. Sin DD Logan AG, Fitzgerald, Liu PP, Bradley TD. Effects of continuous airway pressure on cardiovascular outcomes in heart failure patients with and without Cheyne-Stokes respiration. Circulation 2000; 102: 61-6.

10. Task Force of the European Society of Cardiology and the North American Society of Pacing and Electrophysiology. Heart Rate Variability. Standards of measurement, physiological interpretation and clinical use. Circulation 1996; 93. 1043-1065.

11. La Rovere MT, Bigger JT Jr, Marcus F, Mortara A, Schwartz PJ for the ATRAMI (Autonomic Tone and Reflexes After Myocardial Infarction) Investigators. Baroreflex Sensitivity and heart rate variability in prediction of total cardiac mortality after myocardial infarction. Lancet 1998; 351: 478-484.

12. La Rovere MT, Pinna GD, Maestri R, Mortara A, Capomolla S, Febo O, Ferrari R, Franchini M, Gnemmi M, Opasich C, Riccardi PG, Traversi E, Cobelli F. Short-term heart rate variability strongly predicts sudden cardiac death in chronic heart failure patients. Circulation 2003; 107: 565-70.

\section{TELEMONITORAGGIO DOMESTICO DI SEGNI VITALI E CARDIORESPIRATORI: LA SFIDA DEL PROGETTO HHH}

Gian Domenico Pinna, Roberto Maestri, Soccorso Capomolla

IRCCS Fondazione Salvatore Maugeri, Istituto Scientifico di Montescano $(P V)$

E-mail:gdpinna@fsm.it

\section{Introduzione}

Lo scompenso cardiaco cronico (SCC) è un problema di salute pubblica di crescente gravità che affligge circa 1'1\% degli abitanti dei paesi industrializzati, percentuale che arriva al 5\% nei soggetti anziani. Nonostante i progressi nel trattamento farmacologico, lo scompenso è ancora caratterizzato da un alto tasso di mortalità e da frequenti ricoveri ospedalieri. ${ }^{1}$

Dal momento che solitamente l'ospedalizzazione costituisce lo stadio finale di un processo di instabilizzazione clinica, una identificazione precoce dei segnali di questa instabilizzazione potrebbe consentire un tempestivo intervento terapeutico atto a ristabilire le condizioni del paziente. Quindi, il telemonitoraggio domestico periodico di opportuni parametri clinici e físiologici, unito ad una appropriata gestione medico-infermieristica, potrebbe costituire una efficace ed economicamente valida soluzione. Per quanto riguarda la scelta di questi indicatori di instabilizzazione clinica, oltre ai parametri standard come peso, pressione arteriosa, frequenza cardiaca e sintomi, un aspetto nuovo e totalmente inesplorato è costituito dal monitoraggio di segnali cardio-respiratori registrati nelle 24 ore. Il razionale per un approccio di questo tipo è basato sul fatto che una elevata percentuale (51-70\%) di pazienti con SCC soffre di disturbi respiratori notturni (apnee centrali o ostruttive) e che studi recenti hanno mostrato che sia i disturbi del pattern respiratorio che una variabilità dalla frequenza cardiaca ridotta sono marker di cattiva prognosi. 2,3

Lo studio finanziato della Unione Europea $\mathrm{HHH}$ (Home or Hospital in Heart failure), ha come obiettivo la comparazione dell'efficacia di diverse strategie di telemonitoraggio domestico nella riduzione delle ospedalizzazioni e nel miglioramento della qualità della vita. Le 3 strategie considerate, sono costituite da: a) semplice contatto telefonico mensile (Strategia 1), b) come a) con monitoraggio settimanale di segni vitali (Strategia 2), c) come b) con monitoraggio mensile di registrazioni 24 ore di segnali cardio-respiratori (Strategia 3). Lo studio coinvolge 11 Centri di Reclutamento distribuiti su 3 nazioni: Italia, Polonia e Gran Bretagna. I pazienti arruolati nelle diverse strategie sono complessivamente 450 , con un follow-up di un anno. 


\section{Materiali e Metodi}

Nel progetto dell'architettura dello studio $\mathrm{HHH}$, si è tenuto conto di due specifiche fondamentali: il sistema doveva essere estremamente semplice per i pazienti (una gran parte della popolazione studiata era composta da soggetti con più di 65 anni) ed i costi di gestione estremamente ridotti. Di conseguenza tutti i dispositivi utilizzati per raccogliere $\mathrm{i}$ dati ed i segnali a casa del paziente e per trasmetterli allo staff medico-infermieristico sono stati sviluppati appositamente in collaborazione con ditte esterne al fine di garantire la massima semplicità. Per ridurre i costi di gestione, invece di un sistema tipo call-center, è stato utilizzato un sistema automatizzato IVR (Interactive Voice Response) in grado di ricevere e raccogliere $\mathrm{i}$ dati inviati dai pazienti a casa ed i loro eventuali messaggi vocali. I segni vitali ricevuti sono gestiti mediante un software appositamente sviluppato che consente di automatizzare al massimo le operazioni dello staff infermieristico. Questo software consente di avere automaticamente le liste di trasmissioni dati non effettuate, le eventuali trasmissioni dati con parametri al di fuori di range di accettabilità stabiliti per ciascun paziente o con variazioni tra un invio ed il successivo superiori ad una soglia preimpostata.

Infine, tutti i dispositivi e lo staff tecnico di supporto al sistema e con funzioni di analisi dei segnali cardio-respiratori sono stati centralizzati in ogni Nazione, creando una sorta di Consorzio tra ospedali a livello nazionale che condividono risorse tecnologiche ed umane, riducendo i costi complessivi.

Le informazioni raccolte nello studio e raccolte nel data-base sono: 1) Dati clinici e demografici (dati raccolti all'arruolamento e nei controlli di follow-up), 2) Segni vitali (dati relativi a peso, frequenza cardiaca, pressione, sintomi ed esami del sangue inviati settimanalmente dai pazienti per telefono), 3) Messaggi vocali, 4) Segnali cardio-respiratori telemonitorati (intervalli RR, segnale di respiro, di posizione e di attività fisica), 5) Parametri derivati dall' analisi dei segnali cardio-respiratori (indici di variabilità della frequenza cardiaca, indici di anormalità del pattern respiratorio), 6) Informazioni sul consumo di risorse (dati necessari per la stima dei costi dell'assistenza e del telemonitoraggio).

Tutti i pazienti possono lasciare messaggi vocali (24 ore su 24) sulla macchina IVR centralizzata: i messaggi vengono poi smistati allo staff di pertinenza. I pazienti in Strategia 2 e 3 inviano settimanalmente i loro segni vitali utilizzando il tastierino numerico dell' apparecchio telefonico, con una procedura automatica gestita dall'IVR. Anche questi segnali vengono reindirizzati verso il centro ospedaliero di pertinenza. I registratori cardio-respiratori usati nello studio (Report-24, FM, Monza) sono in grado di registrare 24 ore di ECG, di respiro (tecnica bioimpedenziometrica), di movimento (accelerometro) e di posizione (in piedi/sdraiato). Il registratore è idoneo ad essere utilizzato dal paziente a casa senza supporto. In figura 1 è mostrato il posizionamento dei tre elettrodi e dei sensori di posizione-movimento. Al termine delle 24 ore, la registrazione si ferma automaticamente, il paziente collega il registratore ad un dispositivo modem dedicato (Smart Modem, Appel Elettronica, Torino) che automaticamente instaura la comunicazione con l'IVR e gestisce l'invio dei dati (figura 2). Nel centro di coordinamento nazionale, i segnali vengono ispezionati per un controllo di qualità e, se i criteri di accettabilità sono soddisfatti, vengono analizzati con un software appositamente sviluppato. L'analisi termina con la produzione di un report inviato automaticamente allo staff medico di pertinenza. In figura 3 e 4 sono mostrate due videate esemplificatrici del software di analisi usato per la variabilità della frequenza cardiaca e del segnale respiratorio.



Figura 1. - Posizionamento degli elettrodi e dei sensori

di posizionamento e movimento.

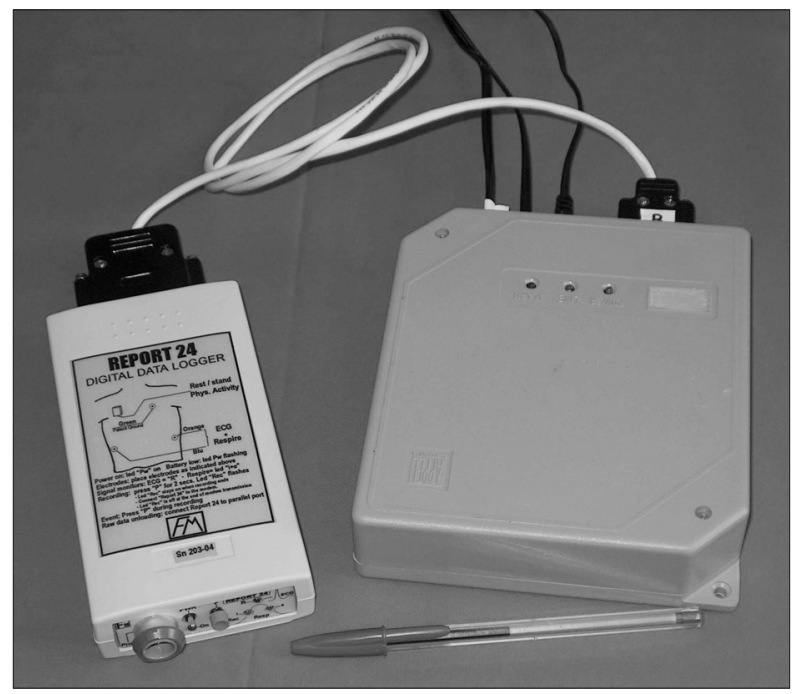

Figura 2. - Il dispositivo
di registrazione dei segnali cardio-respiratori connesso allo Smart Modem.

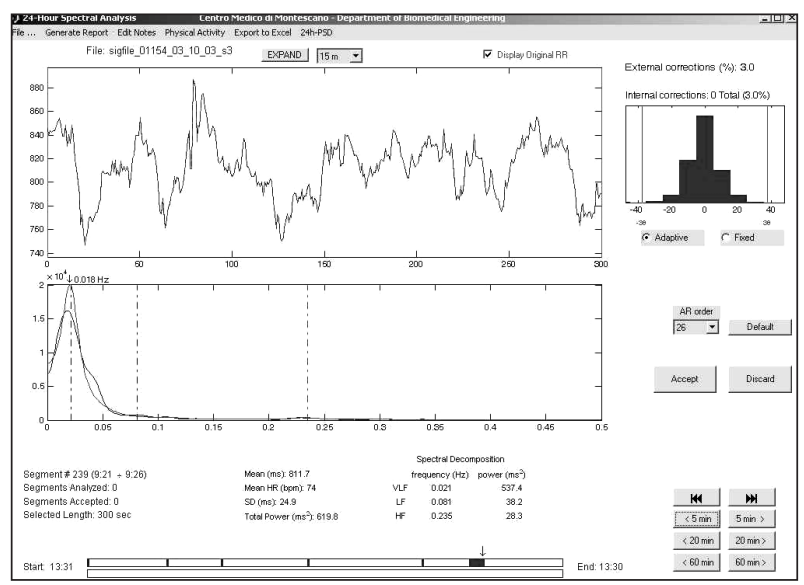

Figura 3. - Videata esemplificativa del software sviluppato per l'analisi della variabilita della frequenza cardiaca.

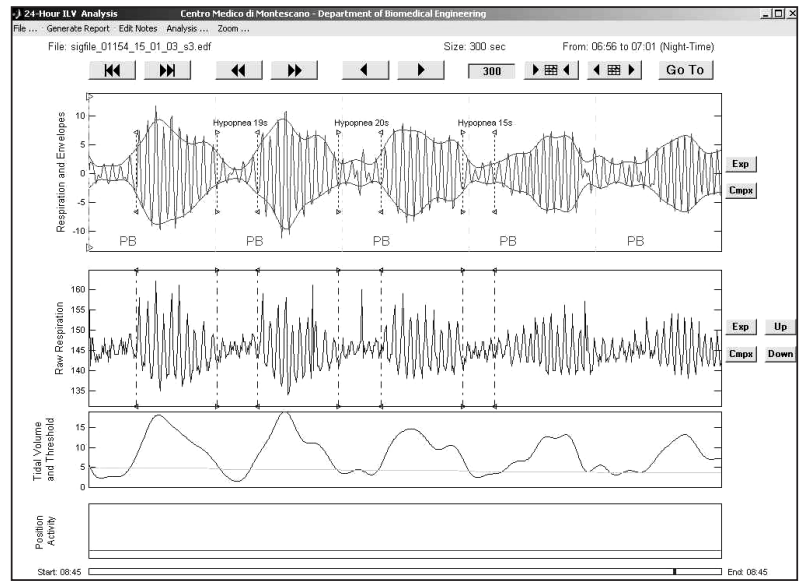

Figura 4 - Videata esemplificativa del software sviluppato per l'analisi delle anomalie del pattern respiratorio. 


\section{Risultati}

Il follow-up dei pazienti arruolati nello studio è terminato nel mese di Agosto 2005.

Per quanto riguarda la fattibilità e la compliance dei pazienti nell'invio dei segni vitali e delle registrazioni cardio-respiratorie vengono riportati i risultati preliminari per l'Italia, ove i pazienti complessivamente arruolati sono 216, di cui 91 coinvolti nell'invio settimanale dei segni vitali e dei segnali cardio-respiratori. Gli invii di segni vitali schedulati e fattibili (paziente vivo e non ospedalizzato) sono stati 4147 , di questi il $71 \%$ sono effettivamente stati effettuati per un totale di 23627 parametri ricevuti. Il numero di esami del sangue trasmessi è di 447 , e sono stati lasciati 335 messaggi vocali. Le trasmissioni mensili di segnali cardio-respiratori programmate e fattibili sono state 831 , ed il $93 \%$ sono state effettuate. Per quanto riguarda la qualità dei segnali trasmessi, (il criterio di eligibilità dello studio impone di avere almeno 2.5 ore durante la notte di segnale respiratorio analizzabile) il $91 \%$ dei segnali ricevuti è risultato idoneo, con una durata del segnale respiratorio analizzabile di $5.8 \pm 1.4$ ore durante la notte.

\section{Conclusioni}

I risultati preliminari dei Centri Italiani del progetto HHH dimostrano chiaramente che sia il telemonitoraggio dei segni vitali sia la registrazione di segnali cardio-respiratori totalmente autogestita dal paziente sono fattibili e la compliance dei pazienti con SCC è molto buona.

Se l'analisi finale dei dati mostrerà che le strategie proposte sono in grado ridurre le ospedalizzazioni e/o gli eventi cardiaci e migliorare la qualità della vita, il modello HHH potrà essere incorporato nella gestione routinaria dello scompenso cardiaco cronico e di altre patologie croniche dove la continuità assistenziale costituisce un fattore importante per le condizioni a lungo termine del paziente.

\section{Bibliografia}

1. Rigel B, Carlson B, Kopp Z, Le Petri B, Glaser D, Unger A. Effect of a standard nurse case management on resource use in patients with chronic heart failure. Arch Intern Med 2002; 162: 705-712.

2. Lanfranchi P, Braghiroli A, Bosimini E, Mazzuero G, Colombo R, Donner CF, Giannuzzi P. Prognostic value of nocturnal Cheyne-Stokes respiration in chronic heart failure patients. Circulation 1999; 99: 1435-1440.

3. La Rovere MT, Pinna GD, Maestri R, Mortara A, Capomolla S, Febo O, Ferrari R, Franchini M, Gnemmi M, Opasich C, Riccardi PG, Traversi E. Short-term heart rate variability predicts sudden cardiac death in chronic heart failure patients. Circulation 2003; 107: $565-570$.

\section{TELECARDIOLOGIA E MEDICINA GENERALE}

\section{Simonetta Scalvini}

\section{Servizio di Telemedicina, IRCCS Fondazione Salvatore Maugeri, Istituto Scientifo di Gussago/Lumezzane (BS) E-mail:sscalvini@fsm.it}

Nell'era della sanità che cambia e nell'era dell'"Information and Communication Technology", la domanda del sistema sanitario per un'assistenza accurata, puntuale ed efficiente, che non si limiti alla sola cura degli episodi acuti, ma tratti la salute come un bene da gestire e conservare nel lungo termine, richiede nuovi approcci al problema. In quest'ottica la telematica, convergenza tra tecnologie informatiche e quelle di telecomunicazione, permette l'abbattimento delle barriere geografiche e può diventare uno strumento fondamentale per la gestione della salute del cittadino.
La telemedicina, intesa come l'applicazione della telematica alla sanità, è in grado di soddisfare il bisogno crescente di soluzioni che mettano in contatto pazienti, medici e competenze specialistiche, indipendentemente dal luogo in cui essi fisicamente si trovano. Tramite un servizio di assistenza remota, pazienti subacuti o cronici possono essere assistiti direttamente a casa loro, con indubbi vantaggi economici e sensibili miglioramenti della qualità di vita percepita.

Recentemente il British Medical Journal ${ }^{1}$ ha indicato, alla luce delle attuali evidenze, quali sono i possibili e quasi ormai indiscussi campi di applicazione della telemedicina: i referti elettronici che alla dimissione dalle strutture di cura dovrebbero essere dati ai pazienti e contemporaneamente inviati a chi ha in carico il paziente stesso (il suo Medico di Medicina Generale - MMG); i consulti per il MMG; l'home telenursing, come ausilio per seguire il paziente affetto da patologie croniche ed i call-center per la salute pubblica.

Lo sviluppo della telemedicina mostra vantaggi per il singolo paziente soprattutto quando si realizza l'interazione tra medicina di base e medicina specialistica. Attraverso i teleconsulti il MMG è in grado di risolvere la maggior parte delle problematiche quotidiane con riduzione della richiesta di accertamenti specialistici, cosa che determina un notevole risparmio economico. Il paziente appare soddisfatto ed il MMG acquisisce competenze; tuttavia ancora numerosi quesiti rimangono aperti. Il passo successivo è l'applicazione di queste possibilità a branche specifiche della medicina. Una recente review ${ }^{2}$ riporta che la migliore applicazione della telemedicina in termini di efficacia è la teleradiologia seguita dalla psichiatria, dalla trasmissione di immagini ecografiche, dalla teledermatologia e dalla telemedicina applicata al paziente cronico in follow-up domiciliare. Tutte queste esperienze sono state eseguite in paesi anglosassoni dove la "primary care" viene condotta con sistemi poco trasferibili alla nostra realtà. La ricerca in telemedicina ha prodotto numerose pubblicazioni che fanno della parte tecnologica un focus centrale, mentre poco è stato pubblicato sulla modellistica e sull'applicabilità dei sistemi di telemedicina alla popolazione generale anche se in quelli editati la soddisfazione del paziente nei confronti del sistema si è rivelata di solito eccellente. 3

Fra le varie esperienze di telemedicina, la telecardiologia ha assunto sicuramente un ruolo di primo piano, poiché le affezioni cardiovascolari sono tra le più rappresentate, soprattutto nella popolazione senile, perché per esse si è ampiamente dimostrata la bontà del rapporto costo/beneficio in termini di vite salvate e recupero funzionale precoce. Da parte degli ospedali vi è la propensione ad evitare ricoveri impropri e ad una precoce dimissione del paziente che quindi ha più spesso necessità di ulteriori cure domiciliari; l'unico riferimento è a questo punto per il paziente il proprio MMG che si trova a gestire situazioni con il solo bagaglio acquisito di esperienze e conoscenze. La telemedicina può in questo caso essere di aiuto all'attività quotidiana professionale dei MMG con il ricorso alle applicazioni telematiche già in uso per il monitoraggio di una serie di parametri vitali quali ecg, saturimetria, pressione arteriosa etc... Con questa metodica il MMG è in grado di selezionare pazienti con cardiopatia nota ad elevato rischio di eventi o pazienti che gli vengono affidati da strutture ospedaliere per un recente ricovero e che sono a rischio di reospedalizzazioni frequenti; questi potrebbero usufruire di sistemi di follow-up telematici che ricomprendano l'educazione sanitaria, la compliance terapeutica, la possibilità di telemonitoraggio e teleassistenza e/o con sistemi di videoconferenza e strutturati attraverso la presenza di un opportuno triage infermieristico e di un collegamento tra MMG e cardiologo specialista o struttura specialistica di riferimento. Esperienze sono riportate in letteratura per alcune patologie 
invalidanti come ad esempio lo scompenso cardiaco cronico.4-9

In letteratura sono riportate alcune esperienze di teleconsulto per i MMG ${ }^{10-12}$ prevalentemente eseguite nei paesi Anglosassoni e del Nord Europa; l'impiego di queste metodiche ha portato a migliorare la motivazione dei pazienti ad avvalersi degli MMG, e a rivolgersi alle strutture specialistiche o ai Dipartimenti di Emergenza solo in caso di reale necessità.

La nostra esperienza Italiana (invio di un tracciato elettrocardiografico 12 derivazioni e teleconsulto cardiologico on-line attraverso un servizio attivo $24 / 24$ ore su tutto il territorio nazionale), vede ad oggi coinvolti più di 1000 MMG sparsi su tutto il territorio nazionale cercando di rispondere ai quesiti suddetti. Il modello applicato ai MMG ha documentato la soluzione in tempo reale dell' $80 \%$ dei problemi cardiologici posti dagli MMG ai Cardiologi risponditori e la presenza di una elevata appropriatezza diagnostica per l'invio alle strutture di emergenza e per la richiesta di approfondimenti diagnostici.1320 È verosimile che gli ottimistici risultati ottenuti siano da attribuire all'efficace teleconsulto che, mediato dall'invio di un tracciato elettrocardiografico, si attiva tra uno specialista cardiologo ed un professionista che conosce in modo approfondito il proprio paziente (anamnesi familiare, remota, fattori di rischio, stile di vita, abitudini voluttuarie, eventuale terapia, etc...) e che quindi orienta e facilita in modo corretto le decisioni finali dello specialista. Attraverso questo servizio riteniamo possibile poter ricostituire un rapporto diretto e fiduciario tra MMG e specialista cardiologo, ottimizzando il loro dialogo clinico e favorendo, in tempo reale, la soluzione di problematiche quotidiane emergenti o routinarie. Attraverso questo rapporto la telecardiologia potrebbe essere utile anche per l'effettiva riduzione degli accessi inutili alle strutture ospedaliere e per la possibilità di ricorrere alla deospedalizzazione di alcune prestazioni diagnostico-terapeutiche.

In una recente review sugli studi riguardanti la reale efficacia in termine di riduzione di costi attraverso la telemedicina ${ }^{16}$ non si è ottenuto un risultato positivo, ma questo appare dovuto alla carenza di studi ben disegnati e condotti; nella nostra esperienza ${ }^{15}$ vi è stato un potenziale risparmio di costi dovuto alla riduzione di circa il $50 \%$ degli accessi alle strutture di emergenza e di circa il $90 \%$ della richiesta di accertamenti diagnostici. Anche in questo caso la preliminarità dello studio ha aperto molti più quesiti rispetto alle risposte date.

Si può infine immaginare che l'assistenza telematica, oggi, debba ricomprendere un network informatico che veda coinvolto il Centro Servizi, i MMG ed i cardiologi di riferimento sia delle strutture ospedaliere sia del territorio, in modo da esaltare le potenzialità del sistema come condivisione di comuni percorsi diagnostico-terapeutici (linee guida), gestione in team dei pazienti sia in acuto sia in cronico, verifica dell'aderenza e compliance terapeutiche, studi di farmacoeconomia, farmacovigilanza ed epidemiologia.

\section{Bibliografia}

1. Wootton R. Telemedicine: Clinical review. BMJ 2001; 323: 55760.

2. Hailay D, Roine R,Ohinmaa A. Systematic review of evidence for the benefits of telemedicine. $J$ Telemed Telecare 2002; 8 (suppl the benefit $1-7$.

3. Mair F, Whitten P. Systematic review of studies of patients satisfaction with telemedicine. BMJ 2000; 320: 1517-1520.

4. Heidenreich PA, Ruggerio CM, Massie BM. Effect of a home monitoring system on hospitalization and resource use for patients with heart failure. Am Heart J 138 (4): 633-640.

5. Bondmass M, Bolger N, Castro G, Avitall B. The effect of Physiologic home monitoring and telemanagement on chronic heart failure outcomes. The Internet Journal of Advanced Nursing Practice 2000; vol. 3: n. 2 .

6. Johnston B, Wheeler L, Deuser J, Sousa KH. Outcomes of the Kaiser Permanente Tele-Home Health Research Project. Arch Fam Med 2000; 9: 40-45.
7. Shah NB, Der E, Ruggerio C, Heidenreich PA, Massie BM Prevention of hospitalisations for heart failure with an interactive home monitoring program. Am Heart J 1998; 135: 373-378.

8. De Lusignan S, Wells S, Johnson P, Meredith K, Leatham E. Compliance and effectiveness of 1 year's home telemonitoring. The report of a pilot study of patient with chronic heart failure. European J of Heart Failure 2001; 3: 723-730.

9. Riegel B, Carlson B, Kopp Z, Le Petri B, Glaser D, Unger A. Effect of a standardised nurse case-management telephone intervention on resource use in patient with chronic heart failure. Arch Inter Med 2002; 162 (6): 705-12.

10. Hallam L, Cragg D. Organization of primary care services outside normal working hours. Br Med J 1994; 1621-1623.

11. Olsen F, Jolleys JV. Out of hours services: the Danish solution examined. Br Med J 1994; 309: 1624-26.

12. Wallace P, Haines A, Harrison R, Barber J, Thompson S, Jacklin P, Roberts J, Lewis L, Wainwright P. Joint teleconsultations (virtual outreach) versus standard outpatient appointments for patients referred by their general practitioner for a specialist opinion: a randomised trial. Lancet 2002; vol. 359 (8): 1961-68.

13. Scalvini S, Zanelli E, Domenighini D, et al. Telecardiology community: a new approach to take care of cardiac patients. Cardiologia 1999; 44 (10): 921-24.

14. Scalvini S, Zanelli E, Gritti M, Pollina R, Giordano A, Glisenti F. Appropriatezza diagnostica dell'invio in pronto soccorso mediante un servizio di telecardiologia. Ital Heart J 2000; 1 (7): 905-909.

15. Scalvini S, Zanelli E, Volterrani M, Castorina M, Giordano A, Glisenti F. Riduzione potenziale dei costi per il Servizio Sanitario Nazionale mediante un servizio di telecardiologia dedicato ai medici di medicina generale. Ital Heart J Suppl 2001: 2 (10): 1091-1097.

16. Scalvini S, Zanelli E, Gritti M, Gazzaniga P, Pollina R, Giordano A, Glisenti F. Telemedicine: utility for care and monitoring in ischemic cardiac disease. Computers in Cardiology 1999; vol. 26: 409-412.

17. Scalvini S, Zanelli E. Telecardiology: a new support for general practitioners in the management of elderly patients. Age and ageing 2002; vol. 31, n. 2 (March 2002): 153.

18. Scalvini S, Zanelli E, Conti C, Volterrani M, Pollina R, Giordano A, Glisenti F. Assessment of prehospital chest pain using telecardiology. Journal of Telemedicine and Telecare 2002; 8, n. 4: 231-236

19. Scalvini S, Piepoli M, Zanelli E, Volterrani M, Giordano A, Glisenti F. Incidence of atrial fibrillation in an italian population followed by their GPS through a telecardiology service. International Journal of Cardiology 2005; vol. 98: 215-220

20. Scalvini S, Vitacca M, Paletta L, Giordano A, Balbi B. Telemedicine: a new frontier for effective healthcare services. Monaldi Archives for chest disease 2004; vol. 61, n. 4 (Pulmonary series): 226-233.

\section{RIABILITAZIONE E MODELLI DI GESTIONE DOMICILIARE DEL PAZIENTE CON SCOMPENSO CARDIACO CRONICO: L'ESPERIENZA DI GUSSAGO}

\author{
Emanuela Zanelli \\ Divisione di Cardiologia Riabilitativa, IRCCS \\ Fondazione Salvatore Maugeri, Istituto Medico \\ di Gussago-Lumezzane (BS) \\ E-mail:czanelli@fsm.it
}

Lo scompenso cardiaco è una sindrome debilitante $\mathrm{e}$ complessa che spesso richiede un approccio specifico per singolo paziente al fine di ottimizzare il trattamento terapeutico ed una serie di interventi psico-sociali e comportamentali.

Gli scarsi risultati raggiunti in termini di miglioramento della salute, nei pazienti con scompenso cardiaco cronico, riflettono il limitato impatto dei farmaci oggi in uso e suggeriscono il bisogno di un regime di trattamento aggiuntivo non farmacologico finalizzato a ridurre le continue reospedalizzazioni che determinano un elevato costo per il sistema sanitario nazionale. Inoltre al diversità e la complessità dei singoli pazienti con scompenso cardiaco cronico impongono, per massimizzare i successi 
clinici l'utilizzo di un approccio multidisciplinare di trattamento.

Negli ultimi 10 anni, sono stati pubblicati numerosi studi che documentano l'efficacia di programmi multidisciplinari domiciliari per la gestione dello scompenso cardiaco cronico. ${ }^{1-4}$

L'approccio integrato deve coinvolgere il paziente, la famiglia, il medico di medicina generale ed i centri cardiologici specializzati. Alla luce quindi di studi osservazionali o randomizzati vi è l'evidenza che l'approccio multidisciplinare per la gestione dello scompenso cardiaco può essere associato ad importanti benefici clinici, comprendenti la riduzione del numero di reospedalizzazioni, il miglioramento della qualità della vita, la soddisfazione del paziente, il miglioramento della tolleranza all'esercizio fisico, l'aumentata capacità di eseguire le normali attività quotidiane e una riduzione generale dei costi.

In taluni studi pubblicati si inizia ad intravedere, all'interno dell'intervento multidisciplinare, un approccio di tipo telematico. L'utilizzo della Telemedicina tuttavia è descritto in modo ancora aneddotico ed in un numero limitato di studi;5-6 per lo più viene realizzato con un approccio telefonico programmato da parte di personale infermieristico e/o medico. Per quanto attiene al monitoraggio transtelefonico questo utilizza la trasmissione di segnali biologici o di variabili fisiologiche attraverso il telefono ad un centro servizi dove un server di rete permette la visualizzazione di questi parametri su personal computer. Lo sviluppo delle tecnologie trasmissive aggiunge a questo metodo efficacia ed efficienza nonché un buon rapporto costo-beneficio.

Il triage infermieristico transtelefonico ha già compiuto 10 anni suscitando grande interesse per la possibilità di utilizzare la Telemedicina come aiuto alla gestione domiciliare dei pazienti, a patto che il personale infermieristico venga opportunamente addestrato ad utilizzare algoritmi computerizzati in modo da indirizzare la conversazione verso determinate e precise conclusioni.7-9 Nell'ambito delle patologie croniche, personale infermieristico, medico di medicina generale e cardiologo possono, in stretta collaborazione, immaginare ed attuare una gestione del paziente con un approccio multidisciplinare ed interdisciplinare. A questo proposito sono stati condotti studi di fattibilità mediante vari tipi di tecnologie $\mathrm{e}$ partendo dal presupposto che i pazienti cronici possono avere beneficio sia utilizzando strumenti semplici che utilizzando tecnologie complesse in sostituzione della tradizionale visita domiciliare. Si tratta generalmente di vari approcci di Telemedicina che, partendo dall'utilizzo delle nuove tecnologie trasmissive, sono stati applicati in studi pilota e di fattibilità.

Sostanzialmente fanno riferimento a due modelli organizzativi:

- il Telenursing in cui la telemedicina è utilizzata nella gestione del paziente con scompenso cardiaco cronico attraverso un sistema di triage infermieristico;

- il Telemanagement, completamente automatizzato, in cui un sistema di telemedicina, mediante messaggi preregistrati ed algoritmi computerizzati, controlla e verifica i segnali vitali del paziente e li rapporta in un range di normalità prestabilito dal medico.

Negli ultimi anni nel nostro centro è stato condotto uno studio pilota in pazienti con scompenso cardiaco cronico in condizioni cliniche stabili e con terapia ottimizzata, con lo scopo di valutare un programma consistente in un follow-up telefonico strutturato ed un monitoraggio elettrocardiografico effettuato da personale medico e paramedico. I pazienti ricevevano un dispositivo portatile in grado di trasmettere, da un telefono fisso o mobile, una monotraccia elettrocardiografia ad un centro servizi; questi era in grado di metterli in comunicazione con perso- nale infermieristico che provvedeva ad un teleconsulto interattivo (chiedendo informazioni riguardanti lo stato di salute, i sintomi, il peso, la diuresi, le modifiche terapeutiche) e forniva informazioni atte ad ottimizzare il trattamento farmacologico ed il trattamento dietetico. Era garantita al paziente l'opportunità di contattare, in qualsiasi momento e per sintomi, il centro servizi (teleassistenza), mentre il team contattava il paziente a date ed orari prestabiliti (telemonitoraggio). ${ }^{10-11}$ Ad un anno di follow-up è stata documentata una riduzione del $56 \%$ delle reospedalizzazioni e del $48 \%$ delle instabilizzazioni (studio caso-controllo)

Per confermare i risultati preliminari raggiunti, è stato redatto e pianificato uno studio prospettico randomizzato multicentrico sulla valutazione costo-beneficio, coinvolgente un più ampio numero di pazienti, con scompenso cardiaco cronico supportato finanziariamente dal Ministero della Salute.

Sono stati arruolati 460 pazienti con scompenso cardiaco cronico (età media $59 \pm 9$ anni), in condizioni stabili e con terapia ottimizzata, di cui 230 nel gruppo HBT (HBT: home based telecardiology) e 230 nel gruppo UC (UC: usual care). Tutti i pazienti sono stati sottoposti all'inizio e alla fine dello studio a visita con esame obiettivo, ecocolordoppler cardiaco, ECG secondo Holter, 6 minuti walking test, questionario Minnesota Living with Heart Failure. Il programma HBT prevedeva un followup transtelefonico con monitoraggio elettrocardiografico eseguito da un team medico e da infermiere professionali (IP) adeguatamente formate alla gestione dello scompenso cardiaco. Ai pazienti veniva affidato un dispositivo portatile in grado di registrare una traccia elettrocardiografica e di trasferirla attraverso un telefono fisso o cellulare ad un centro servizi; qui un IP, disponibile 24/24 ore, provvedeva ad una valutazione della traccia e ad una consulenza interattiva telefonica preordinata e programmata (con rilievo quindi di informazioni sullo stato di salute del paziente, sui sintomi, peso corporeo, diuresi e ottimizzazione terapeutica). I pazienti erano invitati a contattare il centro servizi, spontaneamente, in occasione dell'eventuale dell'insorgenza di sintomi (teleassistenza), mentre il personale infermieristico contattava il paziente seguendo un calendario di appuntamenti prestabiliti (telemonitoraggio).

Nella tabella 1 sono riassunte le caratteristiche delle due popolazioni di pazienti.

Dopo un anno di follow-up, si è evidenziato una riduzione dello score dell'esame obiettivo (da $1.45 \pm 1.57$ a $1.08 \pm 1.33 ; \mathrm{p}<0.05$ ) rispetto al basale che è rimasto invariato (da $1.35 \pm 1.4$ a $1.36 \pm 1.2$; ns); entrambi i gruppi hanno evidenziato un miglioramento del test dei 6 minuti, che è risultato più evidente nel gruppo HBT (HBT $421 \pm 107$ a $452 \pm 97$; $p<0.05$; vs. UC: $388 \pm 90$ a $410 \pm 92$; $\mathrm{p}<0.05)$.

Il gruppo HBT ha evidenziato inoltre un miglioramento del Minnesota Living Questionnaire (29 \pm 20 vs. $23.5 \pm 16.8 \mathrm{p}<0.05)$.

L'analisi degli eventi evidenziati nei due gruppi viene riportata in tabella 2.

La riduzione delle reospedalizzazioni è risultata essere del $38 \%$, rispetto ai pazienti in UC; si è evidenziato inoltre una riduzione della mortalità statisticamente significativa.

Mediante l'invio di una monotraccia elettrocardiografica contestualmente al contatto telefonico, il servizio ha inoltre consentito di escludere, al momento di chiamate per sintomo, aritmie, malfunzionamenti di PM, comparsa di blocchi $\mathrm{A}-\mathrm{V}$ precedentemente non presenti. L'ECG monotraccia è stato inoltre utilizzato nell'implementazione della terapia betabloccante permettendo di incrementare il dosaggio medio di carvedilolo/paziente passando da un dosaggio iniziale medio del carvedilolo di $37.6 \pm 21.5 \mathrm{mg}$ a $41.8 \pm 21.6 \mathrm{mg}$. 
Tabella 1. - Caratteristiche dei gruppi di pazienti con scompenso cardiaco cronico studiati

\begin{tabular}{lccc}
\hline & HBT $(\mathrm{n}=230)$ & $\mathrm{UC}(\mathrm{n}=230)$ & P value \\
\hline Età (anni) & $58 \pm 10$ & $56 \pm 10$ & $\mathrm{~ns}$ \\
Maschi & $193(84 \%)$ & $198(86 \%)$ & $\mathrm{ns}$ \\
NYHA III-IV & $106(46 \%)$ & $70(30.55)$ & 0.005 \\
Eziologia ischemica & $129(56 \%)$ & $106(46 \%)$ & $\mathrm{ns}$ \\
VO$_{2}$ peak (ml/kg/min) & $13.4 \pm 3.4$ & $12.8 \pm 3$ & $\mathrm{~ns}$ \\
Frazione di eiezione (\%) & $28.4 \pm 6.9$ & $26.0 \pm 7.6$ & $\mathrm{~ns}$ \\
Pressione arteriosa sistolica (mmHg) & $107 \pm 14.8$ & $109 \pm 16$ & $\mathrm{~ns}$ \\
Sodio (mEq/L) & $138.7 \pm 3.1$ & $137.7 \pm 3.4$ & $\mathrm{~ns}$ \\
Potassio (mEq/L) & $4.5 \pm 0.5$ & $4.4 \pm 0.4$ & $\mathrm{~ns}$ \\
Creatinina (mg/dl) & $1.38 \pm 0.7$ & $1.37 \pm 0.4$ & $\mathrm{~ns}$ \\
Azoto ureico (mg/dl) & $73.7 \pm 39.4$ & $61.6 \pm 28.3$ & $\mathrm{~ns}$ \\
Farmaci alla dimissione & & & \\
$\quad$ Digossina & $78(34)$ & $115(50)$ & 0.005 \\
$\quad$ Beta-bloccanti & $195(85)$ & $138(60)$ & 0.005 \\
\hline
\end{tabular}

Legenda: HBT: Home Based Telecardiology; UC: Usual Care.

Tabella 2. - Eventi (follow-up 1 anno)

\begin{tabular}{lll}
\hline & Modello UC (n=230) & Modello HBT (n=230) \\
\hline Pz ospedalizzati n (\%) & $78(34)$ & $56(24)$ \\
Rischio Relativo & & 0.62 \\
Limiti di conf. 95\% & & $0.43-0.81$ \\
\hline Pz instabilizz. n. (\%) & $94(41)$ & $60(26)$ \\
Rischio Relativo & & 0.50 \\
Limiti di conf. 95\% & & $0.32-0.68$ \\
\hline Pz deceduti n (\%) & $27(12)$ & $6(6.5)$ \\
Rischio Relativo & & 0.50 \\
Limiti di conf. 95\% & & $0.20-0.80$ \\
\hline
\end{tabular}

Legenda: HBT: Home Based Telecardiology; UC: Usual Care.

Contestualmente è stata eseguita una dettagliata analisi dei costi relativi al sistema che, valorizzando i ricoveri ospedalieri per DRG in vigore in Regione Lombardia, ad un anno di follow-up ma ancora in modo più evidente a 6 mesi, mostra un'evidente risparmio economico dovuto alla riduzione delle reospedalizzazioni.

I nostri dati, seppur preliminari, confermano l'efficacia e la fattibilità di un progetto di telemedicina. Per questa ragione la telemedicina si sta inserendo nei programmi multidisciplinari di gestione dello scompenso cardiaco cronico. Nonostante i trial d'intervento abbiano utilizzato tutti disegni diversi, vi è un unico elemento comune rappresentato dall'infermiera professionale, che appare ave- re un ruolo chiave nel management dello scompenso cardiaco.

Potenziali benefici ottenibili dalla telemedicina si concentrano nella possibilità di erogare una consulenza specialistica al di fuori della struttura ospedaliera, migliorando l'appropriatezza dei ricoveri anche presso le strutture d'emergenza. Probabilmente il risultato cui tendere, coinvolgendo paziente, famiglia, medico di medicina generale e specialista cardiologo, è un approccio integrato che faccia ricorso sia al sistema telematico che alla possibilità di visite domiciliari. Ulteriori studi sono necessari per sviluppare le strategie utili a questo nuovo tipo di management. I risultati ottenuti riguardano il monitoraggio per un breve periodo di tempo, rimanendo incerto l'effetto sul lungo periodo. Se l'analisi costo-beneficio proverà i vantaggi di questi approcci, potremmo immaginare un network informatico che, unendo pazienti, medici di medicina generale, specialisti ospedalieri e dei servizi territoriali, modifichi ed ottimizzi il management territoriale dello scompenso cardiaco cronico migliorando nel contempo la qualità della vita dei pazienti e riducendo i costi sanitari dell'assistenza.

\section{Bibliografia}

1. Rich MW, Beckham V, Wittemburg C, et al. A multidisciplinary intervention to prevent the readmission of elderly participants with congestive heart failure. N Engl J Med 1995; 333: 1190.

2. Stewart S, Marlej JE, Horowitz JD. Effects of a multidisciplinari, home-based intervention among patients with congestive heart failure: a randomised, controlled study. Lancet 1999; 354: 107783.

3. Doughty RN, Wright SP, Pearl A, Walsh HJ, et al. Randomized, controlled trial of integrated heart failure management. The Auckland Heart Failure Management Study. Eur Heart J 2002; 23: $139-146$

4. Shah NB, Der E, Ruggerio C, Heidenreich PA, Massie BM. Prevention of hospitalisations for heart failure with an interactive home monitoring program. Am Heart J 1998; 135: 373-378.

5. De Lusignan S, Wells S, Johnson P, Meredith K, Leatham E. compliance and effectiveness of 1 year's home telemonitoring. The report of a pilot study of patient with chronic heart failure. European J of Heart Failure 2001; 3: 723-730.

6. Jenkins RL, McSweeney M. Assessing elderly patients with congestive heart failure via in- home interactive telecommunication. J Gerontol Nurs 2001; 27 (1): 21-7.

7. Riegel B, Carlson B, Kopp Z, Le Petri B, Glaser D, Unger A. Effect of a standardised nurse case-management telephone intervention on resource use in patient with chronic heart failure. Arch Inter Med 2002; 162 (6): 705-12.

8. Nurse telephone-triage. The Lancet 2001; vol 357, n. 9253: 323.

9. Whitten P, Collins B, Mair F. Nurse and patient reactions to a developmental home telecare system. J Telemed Telecare 1998; 4: 152-160.

10. Martinelli G, Baratti D, Scalvini S, et al. Gestione infermieristica domiciliare mediante un servizio di telecardiologia in pazienti con scompenso cardiaco cronico. IHJ 2001; Vol 2 Suppl 2:172 (abstract).

11. Scalvini S, Zanelli E, Volterrani M, Buscaya O, Domeneghini D, Glisenti F., Giordano A. Effetti di un programma di assistenza domiciliare, mediante telecardiologia, sugli eventi nei pazienti con scompenso cardiaco cronico: uno studio pilota caso-controllo. Ital Heart J 2002; vol. 3 Suppl 2: 141S.

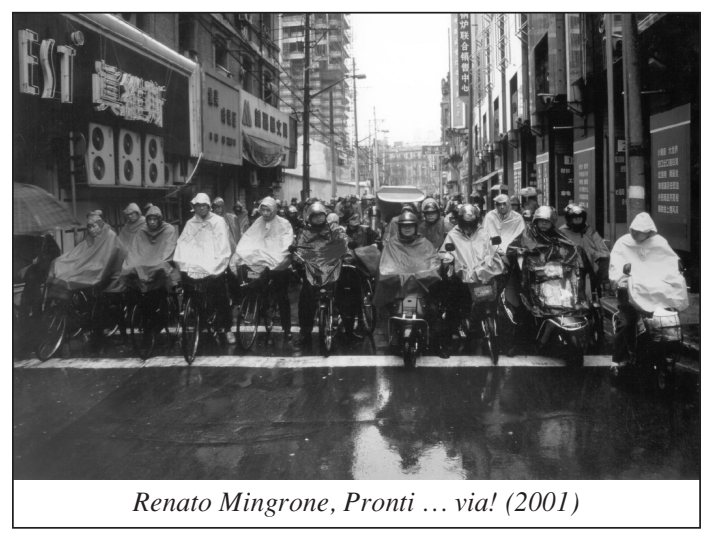

